\title{
ON POMPEIU-CHEBYSHEV FUNCTIONAL AND ITS GENERALIZATION
}

\author{
MOHAMMAD W. ALOMARI
}

\begin{abstract}
In this work, a generalization of Chebyshev functional is presented. New inequalities of Grüss type via Pompeiu's mean value theorem are established. Improvements of some old inequalities are proved. A generalization of pre-Grüss inequality is elaborated. Some remarks to further generalization of Chebyshev functional are presented. As applications, bounds for the reverse of CBS inequality are deduced. Hardy type inequalities on bounded real interval $[a, b]$ under some other circumstances are introduced. Other related ramified inequalities for differentiable functions are also given.
\end{abstract}

\section{INTRODUCTION}

The difference

$$
\mathcal{T}(f, g)=\frac{1}{b-a} \int_{a}^{b} f(t) g(t) d t-\frac{1}{b-a} \int_{a}^{b} f(t) d t \cdot \frac{1}{b-a} \int_{a}^{b} g(t) d t .
$$

is called 'the Chebyshev functional' which it has multiple applications in several mathematical branches specially in Numerical integrations and Probability Theory. For more detailed history see [22].

The most famous bounds of the Chebyshev functional are incorporated in the following theorem:

Theorem 1. Let $f, g:[c, d] \rightarrow \mathbb{R}$ be two absolutely continuous functions, then

$$
\begin{aligned}
& |\mathcal{T}(f, g)| \\
& \leq\left\{\begin{array}{l}
\frac{(d-c)^{2}}{12}\left\|f^{\prime}\right\|_{\infty}\left\|g^{\prime}\right\|_{\infty}, \quad \text { if } f^{\prime}, g^{\prime} \in L_{\infty}([c, d]), \quad \text { proved in [7] } \\
\frac{1}{4}\left(M_{1}-m_{1}\right)\left(M_{2}-m_{2}\right), \quad \text { if } m_{1} \leq f \leq M_{1}, \quad m_{2} \leq g \leq M_{2}, \text { proved in [11] } \\
\frac{(d-c)}{\pi^{2}}\left\|f^{\prime}\right\|_{2}\left\|g^{\prime}\right\|_{2}, \quad \text { if } f^{\prime}, g^{\prime} \in L_{2}([c, d]), \quad \text { proved in [19] } \\
\frac{1}{8}(d-c)(M-m)\left\|h_{2}^{\prime}\right\|_{\infty}, \quad \text { if } m \leq f \leq M, g^{\prime} \in L_{\infty}([c, d]), \text { proved in [23] }
\end{array}\right.
\end{aligned}
$$

The constants $\frac{1}{12}, \frac{1}{4}, \frac{1}{\pi^{2}}$ and $\frac{1}{8}$ are the best possible.

The first inequality in (1.2), is well known as Chebyshev inequality (sometimes called Chebyshev second inequality) which deals with differentiable functions whose first derivatives are bounded. The second inequality in (1.2) is called the Grüss inequality which deals with integrable bounded functions.

Date: August 24, 2021.

2010 Mathematics Subject Classification. 26D15, 26D10, $26 \mathrm{~A} 48$.

Key words and phrases. Chebyshev functional, Grüss inequality, Pompeiu MVT, CBS inequality, Hardy inequality. 
Far away from this, Pompeiu in [26] established a variant Mean Value Theorem (MVT) for real functions defined on a real interval that does not include ' 0 '; nowadays known as Pompeiu's mean value theorem (PMVT), which states that:

Theorem 2. For every real valued function $f$ differentiable on an interval $[a, b]$ not containing 0 and for all pairs $x_{1} \neq x_{2}$ in $[a, b]$ there exists a point $\xi \in\left(x_{1}, x_{2}\right)$ such that

$$
\frac{x_{1} f\left(x_{2}\right)-x_{2} f\left(x_{1}\right)}{x_{1}-x_{2}}=f(\xi)-\xi f^{\prime}(\xi) .
$$

The geometrical interpretation of this theorem as given in [28]: the tangent at the point $(\xi, f(\xi))$ intersects the $y$-axis at the same point as the secant line connecting the points $\left(x_{1}, f\left(x_{1}\right)\right)$ and $\left(x_{2}, f\left(x_{2}\right)\right)$. The proof of PMVT can be done by applying the generalized MVT for derivatives on the functions $x \mapsto \frac{f(x)}{x}$ and $x \mapsto \frac{1}{x}$, where $f$ is differentiable on $[a, b]$.

In 2005, and in viewing of PMVT, Pachpatte [24] proposed the following corresponding Chebyshev functional: For continuous functions $p, q:[a, b] \rightarrow \mathbb{R}$ which are differentiable on $(a, b)$, define

$$
\mathcal{P}(p, q)=\int_{a}^{b} p(x) q(x) d x-\frac{3}{b^{3}-a^{3}}\left(\int_{a}^{b} x p(x) d x\right)\left(\int_{a}^{b} x q(x) d x\right),
$$

and let us call it "Pompeiu-Chebyshev functional". In [24], we find the following result:

Theorem 3. Let $0 \notin[a, b]$. Let $f, g:[a, b] \rightarrow \mathbb{R}$ be two continuous functions on $[a, b]$ and differentiable on $(a, b)$. Then

$$
|\mathcal{P}(p, q)| \leq(b-a)\left(1-\frac{3}{4} \cdot \frac{(a+b)^{2}}{a^{2}+a b+b^{2}}\right)\left\|f-\ell f^{\prime}\right\|_{\infty}\left\|g-\ell g^{\prime}\right\|_{\infty},
$$

where $\ell(t)=t$, for all $t \in[a, b]$.

In the same year, Pečarić and Ungar [25] studied the Pompeiu-Chebyshev functional (1.4), and they obtained the following result.

Theorem 4. Let the functions $f, g:[a, b] \rightarrow \mathbb{R}$ be continuous on $[a, b]$ and differentiable on $(a, b)$ with $0<a<b$. Then for $\frac{1}{p}+\frac{1}{q}=1$, with $1 \leq p, q \leq \infty$, the inequality

$$
\begin{aligned}
|\mathcal{P}(f, g)| \leq \frac{(b-a)^{\frac{1}{p}}}{\left(b^{2}-a^{2}\right)}\left[\left\|f-\ell f^{\prime}\right\|_{p} \int_{a}^{b} x|g(x)|\right. & A(x, p) d x \\
& \left.+\left\|g-\ell g^{\prime}\right\|_{p} \int_{a}^{b} x|f(x)| A(x, p) d x\right]
\end{aligned}
$$

holds, where $\ell(t)=t, t \in[a, b]$ and

$$
\begin{aligned}
A(x, p)=\left(\frac{a^{2-q}-x^{2-q}}{(1-2 q)(2-q)}+\frac{x^{2-q}-a^{1+q} x^{1-2 q}}{(1-2 q)(1+q)}\right)^{\frac{1}{q}} & \\
& +\left(\frac{b^{2-q}-x^{2-q}}{(1-2 q)(2-q)}+\frac{x^{2-q}-b^{1+q} x^{1-2 q}}{(1-2 q)(1+q)}\right)^{\frac{1}{q}},
\end{aligned}
$$

for all $x \in[a, b]$. 
Remark 1. In particular, we have the following special cases in (1.6):

$$
\begin{aligned}
& A(x, \infty)=\frac{a^{2}+b^{2}}{2 x}+x-(a+b), \\
& A(x, 1)=\frac{1}{a}+\frac{b}{x^{2}} \\
& A(x, 2)=\frac{1}{3}\left[\left(\ln \left(\frac{x}{a}\right)^{3}+\frac{a^{3}}{x^{3}}-1\right)^{\frac{1}{2}}+\left(\ln \left(\frac{x}{b}\right)^{3}+\frac{b^{3}}{x^{3}}-1\right)^{\frac{1}{2}}\right],
\end{aligned}
$$

for all $x \in[a, b]$.

In 2015, Dragomir [9] studied the Pompeiu-Chebyshev functional (1.4) with a slightly different consideration. Namely, Dragomir considered (1.4) as

$$
\widehat{\mathcal{P}}(f, g)=\frac{b^{3}-a^{3}}{3} \cdot \mathcal{P}(f, g) .
$$

The following lemma plays a main role in the proof of Dragomir results in [9].

Lemma 1. Let $f:[a, b] \rightarrow \mathbb{C}$ be an absolutely continuous function on the interval $[a, b]$ with $b>a>0$. Then for any $t, x \in[a, b]$, we have

$$
\begin{aligned}
& |t f(x)-x f(t)| \\
& \leq \begin{cases}\left\|f-\ell f^{\prime}\right\|_{\infty}|x-t|, & \text { if } f-\ell f^{\prime} \in L^{\infty}[a, b] \\
\left(\frac{1}{2 q-1}\right)^{\frac{1}{q}}\left\|f-\ell f^{\prime}\right\|_{p}\left|\frac{x^{q}}{t^{q-1}}-\frac{t^{q}}{x^{q-1}}\right|^{\frac{1}{q}}, & \text { if } f-\ell f^{\prime} \in L^{p}[a, b] \\
\left\|f-\ell f^{\prime}\right\|_{1} \frac{\max \{x, t\}}{\min \{x, t\}}, & \text { if } f-\ell f^{\prime} \in L^{1}[a, b]\end{cases}
\end{aligned}
$$

where $p, q>1$ with $\frac{1}{p}+\frac{1}{q}=1$.

The main results in [9], are combined in the following theorem.

Theorem 1. Let $f, g:[a, b] \rightarrow \mathbb{C}$ be absolutely continuous functions on the interval $[a, b]$ with $b>a>0$.

(1) If $f^{\prime}, g^{\prime} \in L^{\infty}[a, b]$, then

$$
|\widehat{\mathcal{P}}(f, g)| \leq \frac{(b-a)^{4}}{12}\left\|f-\ell f^{\prime}\right\|_{\infty}\left\|g-\ell g^{\prime}\right\|_{\infty} .
$$

The constant $\frac{1}{12}$ is the best possible.

(2) If $f^{\prime} \in L^{p}$ and $g^{\prime} \in L^{q}[a, b], p, q>1, p \neq 2 \neq q$ and $\frac{1}{p}+\frac{1}{q}=1$, then

$$
|\widehat{\mathcal{P}}(f, g)| \leq \frac{M_{p}^{\frac{1}{p}}(a, b) M_{q}^{\frac{1}{q}}(a, b)}{2(2 p-1)^{\frac{1}{p}}(2 q-1)^{\frac{1}{q}}}\left\|f-\ell f^{\prime}\right\|_{p}\left\|g-\ell g^{\prime}\right\|_{q},
$$

where

$$
M_{r}(a, b):=\int_{a}^{b} \int_{a}^{b}\left|\frac{x^{r}}{t^{r-1}}-\frac{t^{r}}{x^{r-1}}\right| d t d x
$$

(3) If $f^{\prime}, g^{\prime} \in L^{2}[a, b]$, then

$$
|\widehat{\mathcal{P}}(f, g)| \leq \frac{1}{9}\left\|f-\ell f^{\prime}\right\|_{2}\left\|g-\ell g^{\prime}\right\|_{2}\left[\left(a^{3}+b^{3}\right) \ln \left(\frac{b}{a}\right)-\frac{2}{3}\left(b^{3}-a^{3}\right)\right],
$$


(4) If $f^{\prime}, g^{\prime} \in L^{1}[a, b]$, then

$$
|\widehat{\mathcal{P}}(f, g)| \leq \frac{(b-a)^{2}}{6 a}(a+2 b)\left\|f-\ell f^{\prime}\right\|_{1}\left\|g-\ell g^{\prime}\right\|_{1},
$$

where $\ell(x)=x, x \in[a, b]$.

Remark 1. It is convenient to remark here that, Dragomir proved the sharpness of (1.8) making use of the constant functions $f(t)=g(t)=1$; which is trivial case. It would be more useful if the sharpness holds for non-trivial functions. For this purpose, we consider $f, g:[a, b] \rightarrow \mathbb{R}$ given by $f(t)=g(t)=c t-1, t \in[a, b], b>a>0$ and $c$ any arbitrary non-zero constant. Simple calculations yield the desired sharpness.

Some other inequalities were introduced in literature by many authors, for recent and related results we refer the reader to [1]-[3], [8]-[10], [20] and [27].

This work is divided into five sections, after this introduction, the second and third sections are devoted to elaborate and investigate some new inequalities of Grüss type via Pompeiu's mean value theorem. Improvements of some old inequalities are also provided. In section 4, generalizations of Grüss type inequalities via Boggio mean value theorem are established. As applications, bounds for the reverse CBS inequality are obtained. In section 5, Using some extracted functionals; new Hardy type inequalities and their generalizations are detected. Some other inequalities for differentiable functions are also given.

\section{The Results}

Let us start with the following results regarding positivity of $\widehat{\mathcal{P}}(f, g)$.

Theorem 2. Let $a, b \in \mathbb{R}$ with $b>a>0$. Let $f, g:[a, b] \subseteq \mathbb{R} \rightarrow \mathbb{R}$ be two Lebesgue integrable functions on $[a, b]$ and satisfy the condition

$$
(y f(x)-x f(y))(x g(y)-y g(x)) \leq 0,
$$

for all $x, y \in[a, b]$. Then

$$
\frac{b^{3}-a^{3}}{3} \int_{a}^{b} f(x) g(x) d x \geq \int_{a}^{b} x f(x) d x \int_{a}^{b} x g(x) d x .
$$

Proof. Let $f$ and $g$ be satisfied the given condition

$$
(y f(x)-x f(y))(x g(y)-y g(x)) \leq 0,
$$

for all $x, y \in[a, b]$, therefore we have

$$
\int_{a}^{b} \int_{a}^{b}(y f(x)-x f(y))(x g(y)-y g(x)) d x d y \leq 0 .
$$

but also, we have

$$
\begin{aligned}
& \frac{1}{2} \int_{a}^{b} \int_{a}^{b}(y f(x)-x f(y))(x g(y)-y g(x)) d x d y \\
& =\frac{1}{2} \int_{a}^{b} \int_{a}^{b}\left\{x y f(x) g(y)-y^{2} f(x) g(x)-x^{2} f(y) g(y)+x y f(y) g(x)\right\} d x d y \\
& =\left(\int_{a}^{b} x f(x) d x\right)\left(\int_{a}^{b} y g(y) d y\right)-\frac{b^{3}-a^{3}}{3} \int_{a}^{b} f(x) g(x) d x \\
& \leq 0
\end{aligned}
$$

which proves the inequality (2.1). 
Corollary 1. Let $a, b \in \mathbb{R}$ with $b>a>0$. Let $f, g:[a, b] \subseteq \mathbb{R} \rightarrow \mathbb{R}$ be two Lebesgue integrable functions on $[a, b]$ and satisfy the condition

$$
(y f(x)-x f(y))(x g(y)-y g(x)) \geq 0,
$$

for all $x, y \in[a, b]$. Then

$$
\frac{b^{3}-a^{3}}{3} \int_{a}^{b} f(x) g(x) d x \leq \int_{a}^{b} x f(x) d x \int_{a}^{b} x g(x) d x .
$$

Remark 2. Let $a, b \in \mathbb{R}$ with $b>a>0$. Let $h:[a, b] \rightarrow \mathbb{R}$ be an increasing on $[a, b]$ then the function $f:[a, b] \rightarrow \mathbb{R}$ given by $f(t)=\frac{h(t)}{t}$ is increasing. So that for any two distinct points $x, y \in[a, b]$ with $x \geq y$, we have $y f(x)-x f(y)=h(x)-h(y) \geq 0$. The reverse observation holds for decreasing function $h$. A generalization of monotonicity and thus the previous two results are given in Section 4.

A pre-Grüss like inequality is incorporated in the following theorem:

Theorem 3. Let $a, b \in \mathbb{R}$ with $b>a>0$. Let $f, g:[a, b] \rightarrow \mathbb{R}$ be two Lebesgue integrable functions then the inequality

$$
|\widehat{\mathcal{P}}(f, g)| \leq|\widehat{\mathcal{P}}(f, f)|^{\frac{1}{2}} \cdot|\widehat{\mathcal{P}}(g, g)|^{\frac{1}{2}}
$$

holds and sharp.

Proof. It is easy to verify that

$$
\widehat{\mathcal{P}}(f, g)=\frac{1}{2} \int_{a}^{b} \int_{a}^{b}(t f(x)-x f(t))(t g(x)-x g(t)) d t d x .
$$

For instance, we observe that

$$
\begin{aligned}
\frac{1}{2} \int_{a}^{b} \int_{a}^{b}(t f(x)-x f(t))^{2} d t d x \\
=\frac{1}{2}\left[\int_{a}^{b} \int_{a}^{b} t^{2} f^{2}(x) d t d x+\int_{a}^{b} \int_{a}^{b} x^{2} f^{2}(t) d t d x\right. \\
\left.\quad-2 \int_{a}^{b} t f(t) d t \int_{a}^{b} x f(x) d x\right] \\
=\frac{1}{2}\left[\frac{b^{3}-a^{3}}{3} \cdot 2 \int_{a}^{b} f^{2}(x) d x-2 \int_{a}^{b} t f(t) d t \int_{a}^{b} x f(x) d x\right] \\
=\frac{b^{3}-a^{3}}{3} \int_{a}^{b} f^{2}(x) d x-\left(\int_{a}^{b} x f(x) d x\right)^{2} \\
=\widehat{\mathcal{P}}(f, f) .
\end{aligned}
$$


Now, using the triangle integral inequality and the Cauchy-Schwarz inequality, we have

$$
\begin{aligned}
& |\widehat{\mathcal{P}}(f, g)| \\
& =\frac{1}{2}\left|\int_{a}^{b} \int_{a}^{b}(t f(x)-x f(t))(t g(x)-x g(t)) d t d x\right| \\
& \leq\left(\frac{1}{2} \int_{a}^{b} \int_{a}^{b}|t f(x)-x f(t)|^{2} d t d x\right)^{\frac{1}{2}}\left(\frac{1}{2} \int_{a}^{b} \int_{a}^{b}|t g(x)-x g(t)|^{2} d t d x\right)^{\frac{1}{2}} \\
& =|\widehat{\mathcal{P}}(f, f)|^{\frac{1}{2}} \cdot|\widehat{\mathcal{P}}(g, g)|^{\frac{1}{2}},
\end{aligned}
$$

as desired. The sharpness follows by considering $f(t)=g(t)=c t-1, t \in[a, b], b>a>0$ and $c$ any arbitrary non-zero constant.

Theorem 4. Let $a, b \in \mathbb{R}$ with $b>a>0$. Let $f, g:[a, b] \rightarrow \mathbb{R}$ be two measurable functions. If there exists real numbers $\Phi, \phi, \gamma, \Gamma$ such that $\phi \leq f(s) \leq \Phi$ and $\gamma \leq g(s) \leq \Gamma$ for all $s \in[a, b]$, then the inequality

$$
|\widehat{\mathcal{P}}(f, g)| \leq \frac{1}{2}(b-a)^{2}(b \Phi-a \phi)(b \Gamma-a \gamma)
$$

holds.

Proof. Since $\gamma \leq g(s) \leq \Gamma$ for all $s \in[a, b]$, then $a \gamma \leq t g(x) \leq b \Gamma$ and $-b \Gamma \leq-x g(t) \leq-a \gamma$ adding the last two inequalities we get that $a \gamma-b \Gamma \leq t g(x)-x g(t) \leq b \Gamma-a \gamma$ or we may write $|\operatorname{tg}(x)-x g(t)| \leq b \Gamma-a \gamma$. Similarly, for $f$ we have $|t f(x)-x f(t)| \leq b \Phi-a \phi$. So that we have

$$
\begin{aligned}
|\widehat{\mathcal{P}}(f, g)| & =\frac{1}{2}\left|\int_{a}^{b} \int_{a}^{b}(t f(x)-x f(t))(t g(x)-x g(t)) d t d x\right| \\
& \leq \frac{1}{2} \int_{a}^{b} \int_{a}^{b}|t f(x)-x f(t)||t g(x)-x g(t)| d t d x \\
& \leq \frac{1}{2}(b-a)^{2}(b \Phi-a \phi)(b \Gamma-a \gamma),
\end{aligned}
$$

which proves the result (2.5).

Theorem 5. Let $a, b \in \mathbb{R}$ with $b>a>0$. Let $f:[a, b] \rightarrow \mathbb{R}$ be a Lebesgue integrable and $g:[a, b] \rightarrow \mathbb{R}$ be a measurable and there exists real numbers $\gamma, \Gamma$ such that $\gamma \leq g(s) \leq \Gamma$ for all $s \in[a, b]$, then the inequality

$$
|\widehat{\mathcal{P}}(f, g)| \leq \frac{1}{\sqrt{2}}(b-a)(b \Gamma-a \gamma) \cdot|\widehat{\mathcal{P}}(f, f)|^{\frac{1}{2}}
$$

holds.

Proof. Since $\gamma \leq g(s) \leq \Gamma$ for all $s \in[a, b]$, then $a \gamma \leq t g(x) \leq b \Gamma$ and $-b \Gamma \leq-x g(t) \leq-a \gamma$ adding the last two inequalities we get that $a \gamma-b \Gamma \leq t g(x)-x g(t) \leq b \Gamma-a \gamma$ or we may write $|\operatorname{tg}(x)-x g(t)| \leq b \Gamma-a \gamma$.

Now since

$$
\widehat{\mathcal{P}}(g, g)=\frac{1}{2} \int_{a}^{b} \int_{a}^{b}|t g(x)-x g(t)|^{2} d t d x
$$


it follows that $\widehat{\mathcal{P}}(g, g) \leq \frac{1}{2}(b \Gamma-a \gamma)^{2}(b-a)^{2}$. Employing (2.3) we get the required result.

An improvement of (2.5) is given in the following theorem.

Theorem 6. Let $a, b \in \mathbb{R}$ with $b>a>0$. Let $f, g:[a, b] \rightarrow \mathbb{R}$ be two measurable functions on $[a, b]$. If there exists real numbers $\Phi, \phi, \gamma, \Gamma$ such that $\phi \leq f(s) \leq \Phi$ and $\gamma \leq g(s) \leq \Gamma$ for all $s \in[a, b]$, that satisfies the condition $\phi \Gamma-\Phi \gamma \neq 0$, then the inequality

$$
\leq \begin{cases}\frac{1}{8}\left(b^{2}-a^{2}\right)^{2}\left(\Gamma^{2}-\gamma^{2}\right)\left(\Phi^{2}-\phi^{2}\right), & \text { If } \Gamma a-\gamma b>0, \Phi a-\phi b>0 \\ \frac{1}{8}\left(b^{2}-a^{2}\right)\left(\Gamma^{2}-\gamma^{2}\right) \cdot\left[(\Phi b-\phi a)^{2}+(\Phi a-\phi b)^{2}\right], & \text { If } \Gamma a-\gamma b>0, \Phi a-\phi b<0 \\ \frac{1}{8}\left(b^{2}-a^{2}\right)\left(\Phi^{2}-\phi^{2}\right)\left[(\Gamma b-\gamma a)^{2}+(\Gamma a-\gamma b)^{2}\right], & \text { If } \Gamma a-\gamma b<0, \Phi a-\phi b>0 \\ \frac{1}{8}\left[(\Gamma b-\gamma a)^{2}+(\Gamma a-\gamma b)^{2}\right] \cdot\left[(\Phi b-\phi a)^{2}+(\Phi a-\phi b)^{2}\right], & \text { If } \Gamma a-\gamma b<0, \Phi a-\phi b<0\end{cases}
$$

holds.

Proof. Since $\gamma \leq g(s) \leq \Gamma$ for all $s \in[a, b]$, then $\operatorname{tg}(x) \leq \Gamma t$, and $-x g(t) \leq-\gamma x$, adding these two inequalities we get that $\operatorname{tg}(x)-x g(t) \leq \Gamma t-\gamma x$. Similarly, for $f$ we have $t f(x)-x f(t) \leq \Phi t-\phi x$. So that we have

$$
\begin{aligned}
|\widehat{\mathcal{P}}(f, g)| & =\frac{1}{2}\left|\int_{a}^{b} \int_{a}^{b}(t f(x)-x f(t))(\operatorname{tg}(x)-x g(t)) d t d x\right| \\
& \leq \frac{1}{2} \int_{a}^{b} \int_{a}^{b}|t f(x)-x f(t)||\operatorname{tg}(x)-x g(t)| d t d x \\
& \leq \frac{1}{2} \int_{a}^{b} \int_{a}^{b}|\Phi t-\phi x||\Gamma t-\gamma x| d t d x .
\end{aligned}
$$

Now, substituting $z=\Gamma t-\gamma x$ and $y=\Phi t-\phi x$, where $\Gamma a-\gamma b \leq z \leq \Gamma b-\gamma a$ and $\Phi a-\phi b \leq y \leq \Phi b-\phi a$. Solving the last two equation with respect to $x$ and $t$ we find that

$$
x=\frac{\Phi z-\Gamma y}{\phi \Gamma-\Phi \gamma}, \quad \text { and } \quad t=\frac{\phi z-\gamma y}{\phi \Gamma-\Phi \gamma} .
$$

Clearly, the Jacobian $J(z, y)=1$, and thus we have

$$
\int_{a}^{b} \int_{a}^{b}|\Phi t-\phi x||\Gamma t-\gamma x| d t d x=\int_{\Phi a-\phi b}^{\Phi b-\phi a} \int_{\Gamma a-\gamma b}^{\Gamma b-\gamma a}|y||z| J(z, y) d z d y .
$$

To evaluate the integral in (2.9), we have the following cases:

Case I: If $\Phi a-\phi b>0$ and $\Gamma a-\gamma b>0$, then

$$
\begin{aligned}
\int_{\Phi a-\phi b}^{\Phi b-\phi a} \int_{\Gamma a-\gamma b}^{\Gamma b-\gamma a}|y||z| d z d y & =\frac{(\Gamma b-\gamma a)^{2}-(\Gamma a-\gamma b)^{2}}{2} \cdot \frac{(\Phi b-\phi a)^{2}-(\Phi a-\phi b)^{2}}{2} \\
& =\frac{1}{4}\left(b^{2}-a^{2}\right)^{2}\left(\Gamma^{2}-\gamma^{2}\right)\left(\Phi^{2}-\phi^{2}\right)
\end{aligned}
$$

substituting (2.10) in (2.8) we get the first inequality in (2.7). 
Case II: If $\Phi a-\phi b<0$ and $\Gamma a-\gamma b>0$, then

$$
\begin{aligned}
\int_{\Phi a-\phi b}^{\Phi b-\phi a} \int_{\Gamma a-\gamma b}^{\Gamma b-\gamma a}|y||z| d z d y & =\frac{(\Gamma b-\gamma a)^{2}-(\Gamma a-\gamma b)^{2}}{2} \cdot \frac{(\Phi b-\phi a)^{2}+(\Phi a-\phi b)^{2}}{2} \\
& =\frac{1}{4}\left(b^{2}-a^{2}\right)\left(\Gamma^{2}-\gamma^{2}\right) \cdot\left[(\Phi b-\phi a)^{2}+(\Phi a-\phi b)^{2}\right],
\end{aligned}
$$

substituting (2.11) in (2.8) we get the fourth inequality in (2.7).

Case III: If $\Phi a-\phi b>0$ and $\Gamma a-\gamma b<0$, then

$$
\begin{aligned}
\int_{\Phi a-\phi b}^{\Phi b-\phi a} \int_{\Gamma a-\gamma b}^{\Gamma b-\gamma a}|y||z| d z d y & =\frac{(\Gamma b-\gamma a)^{2}+(\Gamma a-\gamma b)^{2}}{2} \cdot \frac{(\Phi b-\phi a)^{2}-(\Phi a-\phi b)^{2}}{2} \\
& =\frac{1}{4}\left(b^{2}-a^{2}\right)\left(\Phi^{2}-\phi^{2}\right)\left[(\Gamma b-\gamma a)^{2}+(\Gamma a-\gamma b)^{2}\right],
\end{aligned}
$$

substituting (2.12) in (2.8) we get the third inequality in (2.7).

Case IV: If $\Phi a-\phi b<0$ and $\Gamma a-\gamma b<0$, then

$$
\int_{\Phi a-\phi b}^{\Phi b-\phi a} \int_{\Gamma a-\gamma b}^{\Gamma b-\gamma a}|y||z| d z d y=\frac{(\Gamma b-\gamma a)^{2}+(\Gamma a-\gamma b)^{2}}{2} \cdot \frac{(\Phi b-\phi a)^{2}+(\Phi a-\phi b)^{2}}{2}
$$

substituting (2.13) in (2.8) we get the second inequality in (2.7).

Remark 3. Theorem 6 does not work for two identical functions. In other words, we cannot choose $f=g$ or $f=g$ a.e. on $[a, b]$. The reason behind this is that, if one chooses $f=g$ then the substitution $z=\Gamma t-\gamma x$ and $y=\Phi t-\phi x$, will be the same and so that we have $\phi \Gamma-\Phi \gamma=\phi \Phi-\Phi \phi=0$ and this contradicts the assumption that $\phi \Gamma-\Phi \gamma \neq 0$.

More general and extensive case can be done through the following improvement of (2.6).

Theorem 7. Under the assumptions of Theorem 5, then the inequality

$$
|\widehat{\mathcal{P}}(f, g)| \leq \frac{1}{2 \sqrt{3}}|\widehat{\mathcal{P}}(f, f)|^{\frac{1}{2}}\left[2\left(b^{3}-a^{3}\right)(b-a)\left(\Gamma^{2}+\gamma^{2}\right)-3 \Gamma \gamma\left(b^{2}-a^{2}\right)^{2}\right]^{\frac{1}{2}}
$$

holds.

Proof. From Theorem 3, we can state the following

$$
\begin{aligned}
& |\widehat{\mathcal{P}}(f, g)| \\
& =\frac{1}{2}\left|\int_{a}^{b} \int_{a}^{b}(t f(x)-x f(t))(t g(x)-x g(t)) d t d x\right| \\
& \leq\left(\frac{1}{2} \int_{a}^{b} \int_{a}^{b}|t f(x)-x f(t)|^{2} d t d x\right)^{\frac{1}{2}}\left(\frac{1}{2} \int_{a}^{b} \int_{a}^{b}|t g(x)-x g(t)|^{2} d t d x\right)^{\frac{1}{2}} \\
& \leq|\widehat{\mathcal{P}}(f, f)|^{\frac{1}{2}}\left(\frac{1}{2} \int_{a}^{b} \int_{a}^{b}|\Gamma t-\gamma x|^{2} d t d x\right)^{\frac{1}{2}} \\
& \leq|\widehat{\mathcal{P}}(f, f)|^{\frac{1}{2}}\left(\frac{1}{12}\left[2\left(b^{3}-a^{3}\right)(b-a)\left(\Gamma^{2}+\gamma^{2}\right)-3 \Gamma \gamma\left(b^{2}-a^{2}\right)^{2}\right]\right)^{\frac{1}{2}},
\end{aligned}
$$


which gives the desired result in (2.14).

Remark 4. In Theorem 7, if $g=f$ then

$$
|\widehat{\mathcal{P}}(g, g)| \leq \frac{1}{12}\left[2\left(b^{3}-a^{3}\right)(b-a)\left(\Gamma^{2}+\gamma^{2}\right)-3 \Gamma \gamma\left(b^{2}-a^{2}\right)^{2}\right] .
$$

So that, if there exist $\phi \leq f(x) \leq \Phi$, for all $x \in[a, b]$, then

$$
\begin{aligned}
|\widehat{\mathcal{P}}(f, g)| \leq|\widehat{\mathcal{P}}(f, f)|^{1 / 2}|\widehat{\mathcal{P}}(g, g)|^{1 / 2} & \leq \frac{1}{12}\left[2\left(b^{3}-a^{3}\right)(b-a)\left(\Gamma^{2}+\gamma^{2}\right)-3 \Gamma \gamma\left(b^{2}-a^{2}\right)^{2}\right]^{1 / 2} \\
& \times\left[2\left(b^{3}-a^{3}\right)(b-a)\left(\Phi^{2}+\phi^{2}\right)-3 \Phi \phi\left(b^{2}-a^{2}\right)^{2}\right]^{1 / 2},
\end{aligned}
$$

which improves and generalizes (2.7).

Next, an improvement of (1.10) can be presented as follows:

Theorem 8. Let $a, b \in \mathbb{R}$ with $b>a>0$. Let $f, g:[a, b] \rightarrow \mathbb{R}$ be two absolutely continuous functions. If $f^{\prime}, g^{\prime} \in L_{2}[a, b]$, then we have

$$
|\widehat{\mathcal{P}}(f, g)| \leq \frac{2}{9 \pi^{2}} \frac{b^{2}}{a^{6}}\left(b^{3}-a^{3}\right)^{2}(b-a) \cdot\left\|\ell f^{\prime}-f\right\|_{2}\left\|\ell g^{\prime}-g\right\|_{2},
$$

where $\ell(t)=t, t \in[a, b]$.

Proof. Since we have

$$
\begin{aligned}
& |\widehat{\mathcal{P}}(f, g)| \\
& \quad \leq\left(\frac{1}{2} \int_{a}^{b} \int_{a}^{b}|t f(x)-x f(t)|^{2} d t d x\right)^{\frac{1}{2}}\left(\frac{1}{2} \int_{a}^{b} \int_{a}^{b}|t g(x)-x g(t)|^{2} d t d x\right)^{\frac{1}{2}} .
\end{aligned}
$$

Let us write

$$
\int_{a}^{b}(t f(x)-x f(t))^{2} d x=t^{2} \int_{a}^{b} x^{2}\left(\frac{f(x)}{x}-\frac{f(t)}{t}\right)^{2} d x
$$

In [21], G. Milovanović and Ž. Milovanović proved the following inequality:

$$
\begin{array}{rl}
\int_{a}^{b} p(s)(F(s)-F(\eta))^{2} & d s \\
\leq & \frac{4}{\pi^{2}}\left[\max \left\{\int_{a}^{\eta} p(s) d s, \int_{\eta}^{b} p(s) d s\right\}\right]^{2} \int_{a}^{b} r(s)\left(F^{\prime}(s)\right)^{2} d s
\end{array}
$$

for any $\eta \in[a, b]$. where $p$ ia positive and continuous on $[a, b]$ with $\int_{a}^{b} p(s) d s<\infty$ and $F$ is absolutely continuous on $(a, b)$ with $\int_{a}^{b} r(s)\left(F^{\prime}(s)\right)^{2} d s<\infty$ and $r(s)=\frac{1}{p(s)}$. The inequality is sharp. 
In viewing of (2.17) and by setting where $F(s)=\frac{f(s)}{s}$ and $p(s)=s^{2}$, with $\eta=t$ for all $s, t \in[a, b]$, with $b>a>0$ in (2.18) we can state that

$$
\begin{aligned}
& \int_{a}^{b}(t f(x)-x f(t))^{2} d x \\
& =t^{2} \int_{a}^{b} x^{2}\left(\frac{f(x)}{x}-\frac{f(t)}{t}\right)^{2} d x \\
& \leq t^{2} \cdot \frac{4}{\pi^{2}}\left[\max \left\{\int_{a}^{t} s^{2} d s, \int_{t}^{b} s^{2} d s\right\}\right]^{2} \int_{a}^{b} \frac{1}{s^{2}}\left(\left(\frac{f(s)}{s}\right)^{\prime}\right)^{2} d s \\
& =t^{2} \cdot \frac{4}{\pi^{2}}\left[\max \left\{\frac{t^{3}-a^{3}}{3}, \frac{b^{3}-t^{3}}{3}\right\}\right]^{2} \int_{a}^{b} \frac{1}{s^{2}}\left(\frac{s f^{\prime}(s)-f(s)}{s^{2}}\right)^{2} d s \\
& =t^{2} \cdot \frac{4}{\pi^{2}}\left[\frac{b^{3}-a^{3}}{6}+\frac{1}{3}\left|t^{3}-\frac{a^{3}+b^{3}}{2}\right|\right]^{2} \cdot \max _{s \in[a, b]}\left\{\frac{1}{s^{6}}\right\} \cdot \int_{a}^{b}\left(s f^{\prime}(s)-f(s)\right)^{2} d s \\
& =t^{2} \cdot \frac{4}{\pi^{2}} \frac{1}{a^{6}}\left[\frac{b^{3}-a^{3}}{6}+\frac{1}{3}\left|t^{3}-\frac{a^{3}+b^{3}}{2}\right|\right]^{2} \cdot\left\|\ell f^{\prime}-f\right\|_{2}^{2} .
\end{aligned}
$$

Integrating w. r. to $t$ over $[a, b]$, we get

$$
\begin{aligned}
& \int_{a}^{b} \int_{a}^{b}(t f(x)-x f(t))^{2} d x d t \\
& \leq \frac{4}{\pi^{2}} \frac{1}{a^{6}}\left\|\ell f^{\prime}-f\right\|_{2}^{2} \cdot \int_{a}^{b} t^{2} \cdot\left[\frac{b^{3}-a^{3}}{6}+\frac{1}{3}\left|t^{3}-\frac{a^{3}+b^{3}}{2}\right|\right]^{2} d t \\
& \leq \frac{4}{\pi^{2}} \frac{1}{a^{6}}\left\|\ell f^{\prime}-f\right\|_{2}^{2} \cdot(b-a) \sup _{t \in[a, b]} t^{2} \cdot\left[\frac{b^{3}-a^{3}}{6}+\frac{1}{3} \mid t^{3}-\frac{a^{3}+b^{3}}{2}\right]^{2} \\
& \leq \frac{4}{\pi^{2}} \frac{1}{a^{6}}\left\|\ell f^{\prime}-f\right\|_{2}^{2} \cdot(b-a) b^{2} \frac{\left(b^{3}-a^{3}\right)^{2}}{9} .
\end{aligned}
$$

Similarly, for $g$ we have

$$
\int_{a}^{b}(t g(x)-x g(t))^{2} d x \leq \frac{4}{\pi^{2}} \frac{1}{a^{6}}\left\|\ell g^{\prime}-g\right\|_{2}^{2} \cdot(b-a) b^{2} \frac{\left(b^{3}-a^{3}\right)^{2}}{9} .
$$

Substituting (2.20) and (2.21) in (2.16) we get the desired result (2.15).

Corollary 2. Let $a, b \in \mathbb{R}$ with $b>a>0$. Let $f:[a, b] \rightarrow \mathbb{R}$ be an absolutely continuous function such that $f^{\prime} \in L_{2}[a, b]$ and $g:[a, b] \rightarrow \mathbb{R}$ is Lebesgue integrable on $[a, b]$, then we have

$$
|\widehat{\mathcal{P}}(f, g)| \leq \frac{\sqrt{2}}{3 \pi} \frac{b}{a^{3}}\left(b^{3}-a^{3}\right)(b-a)^{1 / 2} \cdot\left\|\ell f^{\prime}-f\right\|_{2}|\widehat{\mathcal{P}}(g, g)|^{\frac{1}{2}},
$$

where $\ell(t)=t, t \in[a, b]$.

Proof. The result follows from (2.3) and (2.15).

Corollary 3. Let $a, b \in \mathbb{R}$ with $b>a>0$. Let $a, b \in \mathbb{R}$ with $b>a>0$. Let $f:[a, b] \rightarrow \mathbb{R}$ be an absolutely continuous function such that $f^{\prime} \in L_{2}[a, b]$ and $g:[a, b] \rightarrow \mathbb{R}$ be a measurable 
function such that $\gamma \leq g(s) \leq \Gamma$ for all $s \in[a, b]$ and for some real numbers $\gamma, \Gamma$, then the inequality

$$
|\widehat{\mathcal{P}}(f, g)| \leq \frac{b}{3 \pi a^{3}}(b-a)(b \Gamma-a \gamma)\left(b^{3}-a^{3}\right) \cdot\left\|\ell f^{\prime}-f\right\|_{2}
$$

holds.

Proof. Substituting (2.5) and (2.15) in (2.3), we get the desired result.

An improvement of (2.15) is given in the following theorem.

Corollary 4. Let $a, b \in \mathbb{R}$ with $b>a>0$. Let $f:[a, b] \rightarrow \mathbb{R}$ be two absolutely continuous functions such that $f^{\prime}, g^{\prime} \in L_{2}[a, b]$, then we have

$$
|\widehat{\mathcal{P}}(f, g)| \leq \frac{7}{162 \pi^{2}} \frac{1}{a^{6}}\left(b^{9}-a^{9}\right) \cdot\left\|\ell f^{\prime}-f\right\|_{2}\left\|\ell g^{\prime}-g\right\|_{2},
$$

where $\ell(t)=t, t \in[a, b]$.

Proof. From (2.19), we have

$$
\begin{aligned}
& \int_{a}^{b} \int_{a}^{b}(t f(x)-x f(t))^{2} d x d t \\
& \leq \frac{4}{\pi^{2}} \frac{1}{a^{6}}\left\|\ell f^{\prime}-f\right\|_{2}^{2} \cdot \int_{a}^{b} t^{2} \cdot\left[\frac{b^{3}-a^{3}}{6}+\frac{1}{3}\left|t^{3}-\frac{a^{3}+b^{3}}{2}\right|\right]^{2} d t \\
& \leq \frac{4}{\pi^{2}} \frac{1}{a^{6}}\left\|\ell f^{\prime}-f\right\|_{2}^{2} \cdot\left[\frac{7}{324}\left(b^{9}-a^{9}\right)-\frac{7}{108} a^{3} b^{3}\left(b^{3}-a^{3}\right)\right] \\
& \leq \frac{4}{\pi^{2}} \frac{1}{a^{6}}\left\|\ell f^{\prime}-f\right\|_{2}^{2} \cdot \frac{7}{324}\left(b^{9}-a^{9}\right),
\end{aligned}
$$

and similarly for $g$ we have

$$
\int_{a}^{b} \int_{a}^{b}(\operatorname{tg}(x)-x g(t))^{2} d x d t \leq \frac{4}{\pi^{2}} \frac{1}{a^{6}}\left\|\ell g^{\prime}-g\right\|_{2}^{2} \cdot \frac{7}{324}\left(b^{9}-a^{9}\right) .
$$

Substituting (2.25) and (2.26) in (2.16) we get the desired result (2.24).

Corollary 5. Let $a, b \in \mathbb{R}$ with $b>a>0$. Let $a, b \in \mathbb{R}$ with $b>a>0$. Let $f:[a, b] \rightarrow \mathbb{R}$ be an absolutely continuous function such that $f^{\prime} \in L_{2}[a, b]$ and $g:[a, b] \rightarrow \mathbb{R}$ is Lebesgue integrable on $[a, b]$, then we have

$$
|\widehat{\mathcal{P}}(f, g)| \leq \frac{\sqrt{7}}{9 \sqrt{2} \pi} \frac{1}{a^{3}}\left(b^{9}-a^{9}\right)^{\frac{1}{2}}\left\|\ell f^{\prime}-f\right\|_{2} \cdot|\widehat{\mathcal{P}}(g, g)|^{\frac{1}{2}} .
$$

Corollary 6. Let $a, b \in \mathbb{R}$ with $b>a>0$. Let $f:[a, b] \rightarrow \mathbb{R}$ be an absolutely continuous function such that $f^{\prime} \in L^{2}[a, b]$ and $g:[a, b] \rightarrow \mathbb{R}$ is measurable on $[a, b]$ such that $\gamma \leq$ $g(s) \leq \Gamma$, for some real numbers $\gamma, \Gamma$ and all $s \in[a, b]$, then the inequality

$$
\begin{aligned}
|\widehat{\mathcal{P}}(f, g)| \leq \frac{\sqrt{7}}{18 \sqrt{6} \pi} \frac{1}{a^{3}}\left(b^{9}-a^{9}\right)^{\frac{1}{2}} \cdot\left\|\ell f^{\prime}-f\right\|_{2} & \\
\times & {\left[2\left(b^{3}-a^{3}\right)(b-a)\left(\Gamma^{2}+\gamma^{2}\right)-3 \Gamma \gamma\left(b^{2}-a^{2}\right)^{2}\right]^{\frac{1}{2}} }
\end{aligned}
$$

holds.

Proof. Substituting (2.24) in (2.14), we get the desired result. 


\section{More inequalities}

Theorem 5. Let $a, b \in \mathbb{R}$ with $b>a>0$. Let $f:[a, b] \rightarrow \mathbb{R}$ be an absolutely continuous functions and $g:[a, b] \rightarrow \mathbb{R}$ is Lebesgue integrable on $[a, b]$.

(1) If $f^{\prime} \in L^{\infty}[a, b]$, then

$$
|\widehat{\mathcal{P}}(f, g)| \leq \frac{1}{2}\left\|f-\ell f^{\prime}\right\|_{\infty} \cdot \int_{a}^{b} \int_{a}^{b}|x-t||\operatorname{tg}(x)-x g(t)| d t d x
$$

(2) If $f^{\prime} \in L^{p}[a, b], p>1$ then

$$
|\widehat{\mathcal{P}}(f, g)| \leq \frac{1}{2}\left(\frac{1}{2 q-1}\right)^{\frac{1}{q}}\left\|f-\ell f^{\prime}\right\|_{p} \cdot \int_{a}^{b} \int_{a}^{b}\left|\frac{x^{q}}{t^{q-1}}-\frac{t^{q}}{x^{q-1}}\right|^{\frac{1}{q}}|t g(x)-x g(t)| d t d x .
$$

(3) If $f^{\prime} \in L^{1}[a, b]$, then

$$
|\widehat{\mathcal{P}}(f, g)| \leq \frac{1}{2}\left\|f-\ell f^{\prime}\right\|_{1} \cdot \int_{a}^{b} \int_{a}^{b} \frac{\max \{x, t\}}{\min \{x, t\}}|\operatorname{tg}(x)-x g(t)| d t d x
$$

$\ell(t)=t, t \in[a, b]$.

Proof. It is easy to observe from Lemma 1 that

(1) If $f^{\prime} \in L^{\infty}[a, b]$, then

$$
\begin{aligned}
|\widehat{\mathcal{P}}(f, g)| & =\left|\int_{a}^{b} \int_{a}^{b}(t f(x)-x f(t))(\operatorname{tg}(x)-x g(t)) d t d x\right| \\
& \leq \frac{1}{2} \int_{a}^{b} \int_{a}^{b}|t f(x)-x f(t)||\operatorname{tg}(x)-x g(t)| d t d x \\
& \leq \frac{1}{2}\left\|f-\ell f^{\prime}\right\|_{\infty} \int_{a}^{b} \int_{a}^{b}|x-t||\operatorname{tg}(x)-x g(t)| d t d x,
\end{aligned}
$$

which proves (3.1).

(2) If $f^{\prime} \in L^{p}[a, b], p>1$ then

$$
\begin{aligned}
|\widehat{\mathcal{P}}(f, g)| & =\left|\int_{a}^{b} \int_{a}^{b}(t f(x)-x f(t))(\operatorname{tg}(x)-x g(t)) d t d x\right| \\
& \leq \frac{1}{2} \int_{a}^{b} \int_{a}^{b}|t f(x)-x f(t)||\operatorname{tg}(x)-x g(t)| d t d x \\
& \leq \frac{1}{2}\left(\frac{1}{2 q-1}\right)^{\frac{1}{q}}\left\|f-\ell f^{\prime}\right\|_{p} \cdot \int_{a}^{b} \int_{a}^{b}\left|\frac{x^{q}}{t^{q-1}}-\frac{t^{q}}{x^{q-1}}\right|^{\frac{1}{q}}|\operatorname{tg}(x)-x g(t)| d t d x,
\end{aligned}
$$

for $p, q>1$ with $\frac{1}{p}+\frac{1}{q}=1$, which proves (3.2). 
(3) If $f^{\prime} \in L^{1}[a, b]$, then

$$
\begin{aligned}
|\widehat{\mathcal{P}}(f, g)| & =\left|\int_{a}^{b} \int_{a}^{b}(t f(x)-x f(t))(\operatorname{tg}(x)-x g(t)) d t d x\right| \\
& \leq \frac{1}{2} \int_{a}^{b} \int_{a}^{b}|t f(x)-x f(t)||t g(x)-x g(t)| d t d x \\
& \leq \frac{1}{2}\left\|f-\ell f^{\prime}\right\|_{1} \cdot \int_{a}^{b} \int_{a}^{b} \frac{\max \{x, t\}}{\min \{x, t\}}|\operatorname{tg}(x)-x g(t)| d t d x,
\end{aligned}
$$

which proves (3.3),

and this end the proof of the theorem.

Theorem 9. Let $a, b \in \mathbb{R}$ with $b>a>0$. Let $f:[a, b] \rightarrow \mathbb{R}$ be an absolutely continuous functions such that $f^{\prime} \in L_{\infty}[a, b]$ and $g:[a, b] \rightarrow \mathbb{R}$ is measurable on $[a, b]$ such that $\gamma \leq g(s) \leq \Gamma$, for some real numbers $\gamma, \Gamma$ and all $s \in[a, b]$, then the inequality

$$
|\widehat{\mathcal{P}}(f, g)| \leq \frac{1}{12}(b-a)^{2}\left\|f-\ell f^{\prime}\right\|_{\infty}\left[2\left(b^{3}-a^{3}\right)(b-a)\left(\Gamma^{2}+\gamma^{2}\right)-3 \Gamma \gamma\left(b^{2}-a^{2}\right)^{2}\right]
$$

holds, where $\ell(t)=t, t \in[a, b]$.

Proof. From (1.8), we have

$$
|\widehat{\mathcal{P}}(f, f)| \leq \frac{(b-a)^{4}}{12}\left\|f-\ell f^{\prime}\right\|_{\infty}^{2} .
$$

Substituting in (2.14), we have

$$
\begin{aligned}
|\widehat{\mathcal{P}}(f, g)| & \leq \frac{1}{2 \sqrt{3}}|\widehat{\mathcal{P}}(f, f)|^{\frac{1}{2}}\left[2\left(b^{3}-a^{3}\right)(b-a)\left(\Gamma^{2}+\gamma^{2}\right)-3 \Gamma \gamma\left(b^{2}-a^{2}\right)^{2}\right]^{\frac{1}{2}} \\
& \leq \frac{1}{12}(b-a)^{2}\left\|f-\ell f^{\prime}\right\|_{\infty}\left[2\left(b^{3}-a^{3}\right)(b-a)\left(\Gamma^{2}+\gamma^{2}\right)-3 \Gamma \gamma\left(b^{2}-a^{2}\right)^{2}\right]^{\frac{1}{2}},
\end{aligned}
$$

and this proves the required inequality.

Theorem 10. Let $a, b \in \mathbb{R}$ with $b>a>0$. Let $f, g:[a, b] \rightarrow \mathbb{R}$ be two absolutely continuous functions such that $f^{\prime} \in L_{\infty}[a, b]$ and $g^{\prime} \in L_{1}[a, b]$, then the inequality then we have

$$
|\widehat{\mathcal{P}}(f, g)| \leq \frac{1}{2}\left(b^{2} a-\frac{a^{3}+8 b^{3}}{9}+\frac{2}{3} b^{3} \ln \left(\frac{b}{a}\right)\right)\left\|f-\ell f^{\prime}\right\|_{\infty}\left\|g-\ell g^{\prime}\right\|_{1}
$$

where $\ell(t)=t, t \in[a, b]$.

Proof. Using (1.7), we have

$$
\begin{aligned}
|\widehat{\mathcal{P}}(f, g)| & =\left|\int_{a}^{b} \int_{a}^{b}(t f(x)-x f(t))(\operatorname{tg}(x)-x g(t)) d x d t\right| \\
& \leq \frac{1}{2} \int_{a}^{b} \int_{a}^{b}|t f(x)-x f(t)||\operatorname{tg}(x)-x g(t)| d x d t \\
& \leq \frac{1}{2}\left\|f-\ell f^{\prime}\right\|_{\infty}\left\|g-\ell g^{\prime}\right\|_{1} \int_{a}^{b} \int_{a}^{b}|x-t| \cdot \frac{\max \{x, t\}}{\min \{x, t\}} d x d t .
\end{aligned}
$$


Now,

$$
\begin{aligned}
& \int_{a}^{b} \int_{a}^{b}|x-t| \cdot \frac{\max \{x, t\}}{\min \{x, t\}} d x d t \\
& =\int_{a}^{b}\left[\int_{a}^{t}|x-t| \cdot \frac{\max \{x, t\}}{\min \{x, t\}} d x+\int_{t}^{b}|x-t| \cdot \frac{\max \{x, t\}}{\min \{x, t\}} d x\right] d t \\
& =\int_{a}^{b}\left[\int_{a}^{t}(t-x) \cdot \frac{t}{x} d x+\int_{t}^{b}(x-t) \cdot \frac{x}{t} d x\right] d t \\
& =\int_{a}^{b}\left[t^{2}(\ln (t)-\ln (a))-t(t-a)+\frac{b^{3}}{3 t}-\frac{t^{2}}{3}-\frac{b^{2}}{2}+\frac{t^{2}}{2}\right] d t \\
& =b^{2} a-\frac{a^{3}+8 b^{3}}{9}+\frac{2}{3} b^{3} \ln \left(\frac{b}{a}\right),
\end{aligned}
$$

substituting in (3.6), we get the desired result.

Theorem 11. Let $a, b \in \mathbb{R}$ with $b>a>0$. Let $f:[a, b] \rightarrow \mathbb{R}$ be an absolutely continuous functions such that $f^{\prime} \in L_{1}[a, b]$ and $g:[a, b] \rightarrow \mathbb{R}$ is measurable on $[a, b]$ such that $\gamma \leq$ $g(s) \leq \Gamma$ for some real numbers $\gamma, \Gamma, s \in[a, b]$, then the inequality

$$
\begin{aligned}
& |\widehat{\mathcal{P}}(f, g)| \\
\leq & \frac{1}{6 \sqrt{2}}\left(\frac{2 b^{3}+a^{3}-3 a b^{2}}{a}\right)^{\frac{1}{2}}\left\|f-\ell f^{\prime}\right\|_{1}\left[2\left(b^{3}-a^{3}\right)(b-a)\left(\Gamma^{2}+\gamma^{2}\right)-3 \Gamma \gamma\left(b^{2}-a^{2}\right)^{2}\right]^{\frac{1}{2}}
\end{aligned}
$$

holds, where $\ell(t)=t, t \in[a, b]$.

Proof. From (1.11), we have

$$
|\widehat{\mathcal{P}}(f, f)| \leq \frac{2 b^{3}+a^{3}-3 a b^{2}}{6 a}\left\|f-\ell f^{\prime}\right\|_{1}^{2} .
$$

Substituting in (2.14), we have

$$
\begin{aligned}
|\widehat{\mathcal{P}}(f, g)| \leq & \frac{1}{2 \sqrt{3}}|\widehat{\mathcal{P}}(f, f)|^{\frac{1}{2}}\left[2\left(b^{3}-a^{3}\right)(b-a)\left(\Gamma^{2}+\gamma^{2}\right)-3 \Gamma \gamma\left(b^{2}-a^{2}\right)^{2}\right]^{\frac{1}{2}} \\
\leq & \frac{1}{6 \sqrt{2}}\left(\frac{2 b^{3}+a^{3}-3 a b^{2}}{a}\right)^{\frac{1}{2}}\left\|f-\ell f^{\prime}\right\|_{1} \\
& \quad \times\left[2\left(b^{3}-a^{3}\right)(b-a)\left(\Gamma^{2}+\gamma^{2}\right)-3 \Gamma \gamma\left(b^{2}-a^{2}\right)^{2}\right]^{\frac{1}{2}}
\end{aligned}
$$

and this proves the theorem.

\section{Generalizations, Remarks and Conclusion}

4.1. Boggio MVT. The MVT of Pompeiu was generalized by Boggio [6] in 1947 (see also, p.,92; [28]), where he proved the following generalization of PMVT:

Theorem 6. For every real valued functions $f$ and $h$ differentiable on an interval $[a, b]$ not containing 0 and for all pairs $x_{1} \neq x_{2}$ in $[a, b]$ there exists a point $\xi \in\left(x_{1}, x_{2}\right)$ such that

$$
\frac{h\left(x_{1}\right) f\left(x_{2}\right)-h\left(x_{2}\right) f\left(x_{1}\right)}{h\left(x_{1}\right)-h\left(x_{2}\right)}=f(\xi)-\frac{h(\xi)}{h^{\prime}(\xi)} f^{\prime}(\xi) " .
$$


As we notice, Boggio generalization of PMVT deals with real functions defined on real intervals not containing ' 0 '. The natural question is: Is it necessarily to exclude ' 0 ' from $[a, b]$ in the Boggio generalization?.

The answer is not necessary to exclude ' 0 ' from $[a, b]$. For example, let $h(x)=x^{2}-4 x+4$, $x \in[-1,1]$. Clearly, $h$ is differentiable and $h(x) \neq 0 \neq h^{\prime}(x)$ for all $x \in[-1,1]$ and $0 \in[-1,1]$. The function $h$ satisfies the assumptions of Theorem 6 , for instance, choose $f(x)=x, x \in[-1,1]$. Applying (4.1), for $x_{1}=-1$ and $x_{2}=1$, we get

$$
\frac{9(1)-1(-1)}{9-1}=\frac{5}{4}=\xi-\frac{\xi^{2}-4 \xi+4}{2 \xi-4} \text {. }
$$

Solving for $\xi$, we get $\xi=\frac{1}{2} \in(-1,1)$. But $0 \in[-1,1](?)$ !. Which means that it's not necessary to exclude ' 0 ' from $[a, b]$.

To deal with more large class of functions and intervals, we need to revise Theorem 6 . For more details about several and various type of MVTs and their generalization(s) we refer the reader to [28].

Let $a, b \in \mathbb{R}$, and $I$ be a real interval such that $a, b$ belong to $I^{\circ}$; the interior of $I$ with $a<b$. Let $\mathcal{A}$ be the set of all real intervals $I$ for which neither $h(x)$ nor $h^{\prime}(x)$ is ever zero on $\mathcal{A}$, where $h: I \rightarrow \mathbb{R}$ is a real valued differentiable function. In symbols, we may write

$$
\mathcal{A}:=\left\{[a, b]: a, b \in I^{\circ}, h(x) \neq 0 \neq h^{\prime}(x), \forall x \in[a, b]\right\} .
$$

In what follows, we revise Theorem 6 and present a new independent proof.

Theorem 7. Let $[a, b] \in \mathcal{A}$. For every real valued differentiable functions $f$ and $h$ defined on $[a, b]$ and all distinct pairs $x_{1}, x_{2} \in[a, b]$ with $h\left(x_{1}\right) \neq h\left(x_{2}\right)$, there exists a point $\xi \in\left(x_{1}, x_{2}\right)$ such that (4.1) holds.

Proof. Let $x_{1}, x_{2}$ be any two distinct points in $[a, b]$ with $x_{1}<x_{2}$ and $h\left(x_{1}\right) \neq h\left(x_{2}\right)$. Define the function $F:\left[x_{1}, x_{2}\right] \rightarrow \mathbb{R}$, given by

$$
F(x)=\left[h\left(x_{1}\right)-h\left(x_{2}\right)\right] \frac{f(x)}{h(x)}-\frac{1}{h(x)}\left[h\left(x_{1}\right) f\left(x_{2}\right)-h\left(x_{2}\right) f\left(x_{1}\right)\right] .
$$

Clearly, $F$ is continuous on $\left[x_{1}, x_{2}\right]$, differentiable on $\left(x_{1}, x_{2}\right)$ and

$$
\begin{aligned}
F\left(x_{1}\right) & =\left[h\left(x_{1}\right)-h\left(x_{2}\right)\right] \frac{f\left(x_{1}\right)}{h\left(x_{1}\right)}-\frac{1}{h\left(x_{1}\right)}\left[h\left(x_{1}\right) f\left(x_{2}\right)-h\left(x_{2}\right) f\left(x_{1}\right)\right] \\
& =f\left(x_{1}\right)-f\left(x_{2}\right) \\
& =F\left(x_{2}\right) .
\end{aligned}
$$

Applying Rolle's theorem, there is an $\xi \in\left(x_{1}, x_{2}\right)$ such that $F^{\prime}(\xi)=0$, so that

$$
\begin{aligned}
F^{\prime}(\xi)= & {\left[h\left(x_{1}\right)-h\left(x_{2}\right)\right] \frac{h(\xi) f^{\prime}(\xi)-f(\xi) h^{\prime}(\xi)}{h^{2}(\xi)} } \\
& \quad-\frac{h^{\prime}(\xi)}{h^{2}(\xi)}\left[h\left(x_{1}\right) f\left(x_{2}\right)-h\left(x_{2}\right) f\left(x_{1}\right)\right] \\
= & 0,
\end{aligned}
$$

equivalently we write

$$
\begin{gathered}
{\left[h\left(x_{1}\right)-h\left(x_{2}\right)\right] h(\xi) f^{\prime}(\xi)=h^{\prime}(\xi)\left[h\left(x_{1}\right) f\left(x_{2}\right)-h\left(x_{2}\right) f\left(x_{1}\right)\right]} \\
+\left[h\left(x_{1}\right)-h\left(x_{2}\right)\right] f(\xi) h^{\prime}(\xi),
\end{gathered}
$$


which means that

$$
\frac{h\left(x_{1}\right) f\left(x_{2}\right)-h\left(x_{2}\right) f\left(x_{1}\right)}{h\left(x_{1}\right)-h\left(x_{2}\right)}=f(\xi)-h(\xi) \frac{f^{\prime}(\xi)}{h^{\prime}(\xi)},
$$

and the proof is established.

Remark 5. Let $a, b \in \mathbb{R}$ with $b>a>0$. By setting $h(x)=x$, we refer to the PMVT. More generally, for $h(x)=x^{r}, r \in \mathbb{R}-\{0\}$, then (4.1) becomes

$$
\frac{x_{1}^{r} f\left(x_{2}\right)-x_{2}^{r} f\left(x_{1}\right)}{x_{1}^{r}-x_{2}^{r}}=f(\xi)-\frac{\xi}{r} f^{\prime}(\xi)
$$

For all distinct pairs $x_{1}, x_{2} \in[a, b]$.

This type of MVT was applied to obtain Ostrowski's type inequalities in [1], [2], [10] and [27]. For comprehensive list of results regarding Ostrowski's inequality see the recent survey $[8]$.

4.2. Pompeiu-Chebyshev functional. Let $a, b \in \mathbb{R}, a<b$. Let $f, g, h:[a, b] \rightarrow \mathbb{R}$ be three integrable functions, then the Pompeiu-Chebyshev functional can be introduced such as:

$$
\begin{aligned}
\widehat{\mathcal{P}}_{h}(f, g) & =\int_{a}^{b} h^{2}(x) d x \int_{a}^{b} f(t) g(t) d t-\int_{a}^{b} f(t) h(t) d t \int_{a}^{b} h(x) g(x) d x \\
& =\frac{1}{2} \int_{a}^{b} \int_{a}^{b}(h(x) f(t)-h(t) f(x))(h(x) g(t)-h(t) g(x)) d t d x .
\end{aligned}
$$

If we consider $h(x)=1$, then $\widehat{\mathcal{P}}_{1}(f, g)=(b-a) \mathcal{T}(f, g)$, which is the Chebyshev functional (1.1). Also, if $h(x)=x, x \in[a, b], b>a>0$, then $\widehat{\mathcal{P}}_{x}(f, g)=\widehat{\mathcal{P}}(f, g)$, which is studied in the Sections 2 and 3.

After we proposed $\widehat{\mathcal{P}}_{h}(f, g)$ independently, we noticed that $\widehat{\mathcal{P}}_{h}(f, g)$ could be deduced from more general identity of Andreiéf's (see [22], p.243), which reads: For two continuous functions $f$ and $g$ defined on $[a, b]$, we have the representation:

$$
\begin{aligned}
\int_{a}^{b} & F_{1}(x) F_{2}(x) d x \int_{a}^{b} G_{1}(x) G_{2}(x) d x-\int_{a}^{b} F_{1}(x) G_{2}(x) d x \int_{a}^{b} F_{2}(x) G_{1}(x) d x \\
& =\frac{1}{2} \int_{a}^{b} \int_{a}^{b}\left(F_{1}(x) G_{1}(y)-F_{1}(y) G_{1}(x)\right)\left(F_{2}(x) G_{2}(y)-F_{2}(y) G_{2}(x)\right) d x d y .
\end{aligned}
$$

Simply, substituting $F_{1}(x)=F_{2}(x)=h(x), G_{1}(x)=f(x)$, and $G_{2}(x)=g(x)$ in (4.3), then we obtain (4.2).

Lemma 2. Let $f, h:[a, b] \rightarrow \mathbb{R}$ be two absolutely continuous function on the interval $[a, b]$. Then for any $t, x \in[a, b]$, we have

$$
|h(t) f(x)-h(x) f(t)| \leq|h(x)||h(t)|\left\{\begin{array}{l}
\left|h f^{\prime}-f h^{\prime}\right|_{\infty,[t, x]} \int_{t}^{x} \frac{d s}{|h(s)|^{2}} \\
\left|h f^{\prime}-f h^{\prime}\right|_{p,[t, x]}\left(\int_{t}^{x} \frac{d s}{|h(s)|^{2 q}}\right)^{\frac{1}{q}} \\
\sup _{s \in[t, x]}\left\{\frac{1}{|h(s)|^{2}}\right\}\left|h f^{\prime}-f h^{\prime}\right|_{1,[t, x]}
\end{array}\right.
$$

where $p, q>1$ with $\frac{1}{p}+\frac{1}{q}=1$. Provided that $h(s) \neq 0$, for $s \in[a, b]$. 
Proof. Since $f$ and $h$ are absolutely continuous functions on $[a, b]$, then $\frac{f(s)}{h(s)}$ is absolutely continuous on $[a, b]$ and so that

$$
\int_{t}^{x}\left(\frac{f(s)}{h(s)}\right)^{\prime} d s=\frac{f(x)}{h(x)}-\frac{f(t)}{h(t)}
$$

for any $t, x \in[a, b]$ with $x \neq t$.

Since

$$
\int_{t}^{x}\left(\frac{f(s)}{h(s)}\right)^{\prime} d s=\frac{h(s) f^{\prime}(s)-f(s) h^{\prime}(s)}{h^{2}(s)}
$$

we get the identity

$$
h(t) f(x)-h(x) f(t)=h(x) h(t) \int_{t}^{x} \frac{h(s) f^{\prime}(s)-f(s) h^{\prime}(s)}{h^{2}(s)} d s .
$$

Taking the modulus, we have

$$
\begin{aligned}
& |h(t) f(x)-h(x) f(t)| \\
& =|h(x)||h(t)|\left|\int_{t}^{x} \frac{h(s) f^{\prime}(s)-f(s) h^{\prime}(s)}{h^{2}(s)} d s\right| \\
& \leq|h(x)||h(t)| \int_{t}^{x}\left|\frac{h(s) f^{\prime}(s)-f(s) h^{\prime}(s)}{h^{2}(s)}\right| d s \\
& \leq|h(x)||h(t)|\left\{\begin{array}{l}
\sup _{s \in[t, x]}\left|h(s) f^{\prime}(s)-f(s) h^{\prime}(s)\right| \int_{t}^{x} \frac{d s}{|h(s)|^{2}} \\
\left(\int_{x}^{t}\left|h(s) f^{\prime}(s)-f(s) h^{\prime}(s)\right|^{p} d s\right)^{\frac{1}{p}}\left(\int_{t}^{x} \frac{d s}{|h(s)|^{2 q}}\right)^{\frac{1}{q}}
\end{array}\right. \\
& =|h(x)||h(t)|\left\{\begin{array}{l}
\left|h f^{\prime}-f h^{\prime}\right|_{\infty,[t, x]} \int_{t}^{x} \frac{d s}{|h(s)|^{2}} \\
\sup _{s \in[t, x]}\left\{\frac{1}{|h(s)|^{2}}\right\} \int_{t}^{x}\left|h(s) f^{\prime}(s)-f(s) h^{\prime}(s)\right| d s
\end{array}\right. \\
& \left|h f^{\prime}-f h^{\prime}\right|_{p,[t, x]}\left(\int_{t}^{x} \frac{d s}{|h(s)|^{2 q}}\right)^{\frac{1}{q}}, \\
& \sup _{s \in[t, x]}\left\{\frac{1}{|h(s)|^{2}}\right\}\left|h f^{\prime}-f h^{\prime}\right|_{1,[t, x]}
\end{aligned}
$$

which completes the proof.

Remark 6. In the previous lemma, if we choose $h(s)=s^{r}, r \geq 1, s \in[a, b], b>a>0$. we have

$$
\left|t^{r} f(x)-x^{r} f(t)\right|=\left\{\begin{array}{l}
\frac{1}{2 r-1} \cdot\left|\frac{t^{r}}{x^{r-1}}-\frac{x^{r}}{t^{r-1}}\right|\left|\ell_{r} f^{\prime}-r \ell_{r-1} f\right|_{\infty,[t, x]} \\
\left(\frac{1}{2 r q-1}\right)^{\frac{1}{q}}\left|\frac{t^{r q}}{x^{r q-1}}-\frac{x^{r q}}{t^{r q-1}}\right|^{\frac{1}{q}}\left|\ell_{r} f^{\prime}-r \ell_{r-1} f\right|_{p,[t, x]} \\
\left(\frac{\max \{t, x\}}{\min \{t, x\}}\right)^{r}\left|\ell_{r} f^{\prime}-r \ell_{r-1} f\right|_{1,[t, x]}
\end{array}\right.
$$

for $p, q>1$ with $\frac{1}{p}+\frac{1}{q}=1$, where $\ell_{r}(s)=s^{r}, s \in[a, b]$ and $r \geq 1$. 
Remark 7. By following the same approaches considered in [9] and in the Sections 2 and 3 of this work one can state more general results concerning $\widehat{\mathcal{P}}_{h}(f, g)$. We left this part to the interested reader and focused researchers.

Definition 1. A real valued function $f$ defined on $[a, b]$ is called increasing (decreasing) with respect to a non-negative function $h:[a, b] \rightarrow \mathbb{R}_{+}$or simply $h$-increasing (h-decreasing) if and only if

$$
h(x) f(t)-h(t) f(x) \geq(\leq) 0
$$

whenever $t \geq x$, for every $x, t \in[a, b]$. In special case if $h(x)=1$ we refer to the original monotonicity. Also, if $h(x)=x$ we have

$$
x f(t)-t f(x) \geq(\leq) 0,
$$

which used in Theorem 2.

Next result generalize the inequality (2.1) and the Chebyshev first inequality (see (4.6)):

Theorem 12. Let $a, b \in \mathbb{R}$ with $a<b$. Let $f, g:[a, b] \subseteq \mathbb{R} \rightarrow \mathbb{R}$ be three integrable functions on $[a, b]$. If $h:[a, b] \rightarrow \mathbb{R}_{+}$is integrable on $[a, b]$ and $f$ and $g$ are both $h$-increasing or h-decreasing on $[a, b]$, then

$$
\int_{a}^{b} h^{2}(x) d x \int_{a}^{b} f(x) g(x) d x \geq \int_{a}^{b} f(x) h(x) d x \int_{a}^{b} h(x) g(x) d x .
$$

Proof. Assume If $f$ and $g$ are both $h$-increasing on $[a, b]$, then we have

$$
[h(x) f(y)-h(y) f(x)][h(x) g(y)-h(y) g(x)] \geq 0,
$$

for all $x, y \in[a, b]$, therefore we have

$$
\int_{a}^{b} \int_{a}^{b}[h(x) f(y)-h(y) f(x)][h(x) g(y)-h(y) g(x)] d x d y \geq 0 .
$$

But also we have

$$
\begin{aligned}
& \frac{1}{2} \int_{a}^{b} \int_{a}^{b}[h(x) f(y)-h(y) f(x)][h(x) g(y)-h(y) g(x)] d x d y \\
& =\int_{a}^{b} h^{2}(x) d x \int_{a}^{b} f(x) g(x) d x-\int_{a}^{b} f(x) h(x) d x \int_{a}^{b} h(x) g(x) d x \\
& \geq 0,
\end{aligned}
$$

which proves the inequality (4.5).

Remark 8. In (4.5), if $h(t)=1, t \in[a, b]$, we recapture the first Chebyshev inequality, which reads:

$$
(b-a) \int_{a}^{b} f(x) g(x) d x \geq \int_{a}^{b} f(x) d x \int_{a}^{b} g(x) d x .
$$

If $h(t)=t, t \in[a, b]$, then we recapture (2.1).

Corollary 7. Let $a, b \in \mathbb{R}$ with $a<b$. Let $f, g, h:[a, b] \subseteq \mathbb{R} \rightarrow \mathbb{R}$ be two integrable functions on $[a, b]$. If $h:[a, b] \rightarrow \mathbb{R}_{+}$is integrable on $[a, b], f$ is $h$-increasing and $g$ is $h$-decreasing on $[a, b]$, then

$$
\int_{a}^{b} h^{2}(x) d x \int_{a}^{b} f(x) g(x) d x \leq \int_{a}^{b} f(x) h(x) d x \int_{a}^{b} h(x) g(x) d x .
$$


Proof. The proof goes likewise the proof of Theorem 12 .

A generalization of Theorem 3, the celebrated pre-Grüss inequality is incorporated in the following theorem.

Corollary 8. Let $a, b \in \mathbb{R}$ with $a<b$. Let $f, g:[a, b] \subseteq \mathbb{R} \rightarrow \mathbb{R}$ be two integrable functions on $[a, b]$. If $h:[a, b] \rightarrow \mathbb{R}_{+}$is integrable on $[a, b]$, then

$$
\left|\widehat{\mathcal{P}}_{h}(f, g)\right| \leq\left|\widehat{\mathcal{P}}_{h}(f, f)\right|^{\frac{1}{2}}\left|\widehat{\mathcal{P}}_{h}(g, g)\right|^{\frac{1}{2}}
$$

or equivalently we can write

$$
\left|\widehat{\mathcal{P}}_{h}(f, g)\right| \leq\left|\widehat{\mathcal{P}}_{f}(h, h)\right|^{\frac{1}{2}}\left|\widehat{\mathcal{P}}_{g}(h, h)\right|^{\frac{1}{2}} .
$$

Both inequalities are sharp.

Proof. Since

$$
\left|\widehat{\mathcal{P}}_{h}(f, g)\right|=\left|\frac{1}{2} \int_{a}^{b} \int_{a}^{b}[h(x) f(y)-h(y) f(x)][h(x) g(y)-h(y) g(x)] d x d y\right|
$$

Utilizing the triangle inequality and then the Cauchy-Bunyakovsky-Schwarz (CBS) inequality, we get

$$
\begin{aligned}
\left|\widehat{\mathcal{P}}_{h}(f, g)\right| \leq & \frac{1}{2} \int_{a}^{b} \int_{a}^{b}|h(x) f(y)-h(y) f(x)||h(x) g(y)-h(y) g(x)| d x d y \\
\leq & \left.\frac{1}{2} \int_{a}^{b} \int_{a}^{b}(h(x) f(y)-h(y) f(x))^{2} d y d x\right)^{\frac{1}{2}} \\
& \times\left(\frac{1}{2} \int_{a}^{b} \int_{a}^{b}(h(x) g(y)-h(y) g(x))^{2} d y d x\right)^{\frac{1}{2}} .
\end{aligned}
$$

On the other hand, we have

$$
\begin{aligned}
\frac{1}{2} \int_{a}^{b} \int_{a}^{b}(h(x) f(y)-h(y) f(x))^{2} d y d x & =\int_{a}^{b} h^{2}(x) d x \int_{a}^{b} f^{2}(y) d y-\left(\int_{a}^{b} h(x) f(x) d x\right)^{2} \\
& =\widehat{\mathcal{P}}_{h}(f, f) \equiv \widehat{\mathcal{P}}_{f}(h, h),
\end{aligned}
$$

and similarly,

$$
\begin{aligned}
\frac{1}{2} \int_{a}^{b} \int_{a}^{b}(h(x) g(y)-h(y) g(x))^{2} d y d x & =\int_{a}^{b} h^{2}(x) d x \int_{a}^{b} g^{2}(y) d y-\left(\int_{a}^{b} h(x) g(x) d x\right)^{2} \\
& =\widehat{\mathcal{P}}_{h}(g, g) \equiv \widehat{\mathcal{P}}_{g}(h, h) .
\end{aligned}
$$

Substituting in (4.9), we get the required result.

Remark 9. In (4.8), if $h(t)=1, t \in[a, b]$, we recapture the classical version of pre-Grüss inequality, which reads:

$$
|\mathcal{T}(f, g)| \leq|\mathcal{T}(f, f)|^{\frac{1}{2}}|\mathcal{T}(g, g)|^{\frac{1}{2}} .
$$

If $h(t)=t, t \in[a, b]$, then we recapture (2.3). 
Remark 10. The weighted version of $\widehat{\mathcal{P}}_{h}(f, g)$ can be presented using Andreiéf's weighted version of (4.3) (see [22], p.244), which reads: For arbitrary continuous functions $F_{i}, G_{i}$ : $[a, b] \rightarrow \mathbb{R}(i=1,2)$ defined on $[a, b]$ and a positive continuous function $p:[a, b] \rightarrow \mathbb{R}_{+}$, we have

$$
\begin{aligned}
& \left|\begin{array}{ll}
\int_{a}^{b} p(x) F_{1}(x) G_{1}(x) d x & \int_{a}^{b} p(x) F_{1}(x) G_{2}(x) d x \\
\int_{a}^{b} p(x) F_{2}(x) G_{1}(x) d x & \int_{a}^{b} p(x) F_{2}(x) G_{2}(x) d x
\end{array}\right| \\
& =\frac{1}{2} \int_{a}^{b} \int_{a}^{b}\left|\begin{array}{ll}
F_{1}\left(x_{1}\right) & F_{1}\left(x_{2}\right) \\
F_{2}\left(x_{1}\right) & F_{2}\left(x_{2}\right)
\end{array}\right|\left|\begin{array}{lll}
G_{1}\left(x_{1}\right) & G_{1}\left(x_{2}\right) \\
G_{2}\left(x_{1}\right) & G_{2}\left(x_{2}\right)
\end{array}\right| p\left(x_{1}\right) p\left(x_{2}\right) d x_{1} d x_{2} .
\end{aligned}
$$

For $p(x)=1$, we get the original version of Andreiéf's identity (4.3). Moreover, by substituting $F_{1}(t)=G_{1}(t)=h(t), F_{2}(t)=f(t), G_{2}(t)=g(t)$ and $p \equiv p$ in (4.10), then we obtain the following weighted version of Pompeiu-Chebyshev functional

$$
\begin{aligned}
& \widehat{\mathcal{P}}_{h}(f, g ; p) \\
& =\int_{a}^{b} p(x) h^{2}(x) d x \cdot \int_{a}^{b} p(x) f(x) g(x) d x-\int_{a}^{b} p(x) h(x) g(x) d x \cdot \int_{a}^{b} p(x) h(x) f(x) d x \\
& =\frac{1}{2} \int_{a}^{b} \int_{a}^{b} p\left(x_{1}\right) p\left(x_{2}\right)\left[h\left(x_{1}\right) f\left(x_{2}\right)-h\left(x_{2}\right) f\left(x_{1}\right)\right]\left[h\left(x_{1}\right) g\left(x_{2}\right)-h\left(x_{2}\right) g\left(x_{1}\right)\right] d x_{1} d x_{2} .
\end{aligned}
$$

Remark 11. In his work [9], Dragomir considered the Chebyshev functional $\mathcal{T}\left(F_{1}, F_{2}\right)$ between two absolutely continuous mappings $F_{1}(x)=\frac{f(x)}{x}$ and $F_{2}(x)=\frac{g(x)}{x}, x \in[a, b]$, $b>a>0$. This can be generalized in terms of h-function as mentioned above (see Lemma 2 above, and Theorem 2.1 in [9]). We left this part to the interested reader.

4.3. The reverse of CBS inequality. The Pompeiu-Chebyshev functional $\widehat{\mathcal{P}}_{h}(\cdot, \cdot)$ can be very useful to bound the reverse of CBS inequality, which it has many applications in various branches of Mathematics, Physics and Statistics. In another context, we find the following related result due to Barnett and Dragomir [5]:

$$
\begin{aligned}
& \int_{a}^{b} h^{2}(x) d x \int_{a}^{b} f^{2}(x) d x-\left(\int_{a}^{b} h(x) f(x) d x\right)^{2} \\
& \leq H^{2} \begin{cases}\frac{(b-a)^{2 p+2}}{(2 p+1)(2 p+2)}\|h\|_{\infty}^{4}, & \text { if } h \in L^{\infty}[a, b] \\
\frac{2^{-\frac{1}{\beta}}(b-a)^{2 p+\frac{2}{\alpha}}}{(2 \alpha p+1)^{\frac{1}{\alpha}}(2 \alpha p+2)^{\frac{1}{\alpha}}}\|h\|_{2 \beta}^{4}, & \text { if } h \in L^{2 \beta}[a, b], \\
\frac{1}{2}(b-a)^{2 p}\|h\|_{2}^{4}, & \text { if } h \in L^{2}[a, b]\end{cases}
\end{aligned}
$$

for $\alpha>1, \frac{1}{\alpha}+\frac{1}{\beta}=1$, where $f$ and $h$ are assumed to be measurable on $[a, b]$ and $\frac{f}{h}$ is Hölder continuous of order $p \in(0,1]$ with Hölderian constant $H>0$, and

$$
\|h\|_{\infty}=\operatorname{ess} \sup _{t \in[a, b]}|h(t)|, \text { and }\|h\|_{p}=\left(\int_{a}^{b}|h(t)|^{p} d t\right)^{\frac{1}{p}}, p \geq 1,
$$

are the usual Lebesgue norms. Clearly, this result support our consideration to generalize the Chebyshev functional as presented in the functional $\widehat{\mathcal{P}}_{h}(\cdot, \cdot)$. Moreover, if $p=1$ in 
(4.11), i.e., $\frac{f}{h}$ satisfy the Lipschitz condition we get

$$
\begin{aligned}
\int_{a}^{b} h^{2}(x) d x \int_{a}^{b} f^{2}(x) d x- & \left(\int_{a}^{b} h(x) f(x) d x\right)^{2} \\
& \leq L^{2} \begin{cases}\frac{(b-a)^{4}}{12}\|h\|_{\infty}^{4}, & \text { if } h \in L^{\infty}[a, b] \\
\frac{2^{-\frac{1}{\beta}}(b-a)^{2+\frac{2}{\alpha}}}{(2 \alpha+1)^{\frac{1}{\alpha}}(2 \alpha+2)^{\frac{1}{\alpha}}}\|h\|_{2 \beta}^{4}, & \text { if } h \in L^{2 \beta}[a, b] \\
\frac{1}{2}(b-a)^{2}\|h\|_{2}^{4}, & \text { if } h \in L^{2}[a, b]\end{cases}
\end{aligned}
$$

It was shown in [5] that, the constant $\frac{1}{12}$ is the best possible. This can be seen by choosing $f(x)=x$ and $h(x)=1$.

Fortunately, $\widehat{\mathcal{P}}_{h}(f, f)$ represents (exactly) the revers of CBS inequality, therefore we can state some related results, as follows:

Theorem 8. Assume that $f$ and $g$ are measurable on $[a, b]$ and $\frac{f}{h}, \frac{g}{h}$ are Hölder continuous of order $p, q \in(0,1]$ with Hölderian constants $H_{1}, H_{2}>0$; respectively, then we have

$$
\left|\widehat{\mathcal{P}}_{h}(f, g)\right| \leq H_{1} H_{2} \begin{cases}\frac{(b-a)^{p+q+2}}{\sqrt{(2 p+1)(2 p+2)(2 q+1)(2 q+2)}}\|h\|_{\infty}^{4}, & \text { if } h \in L^{\infty}[a, b] \\ \frac{2^{-\frac{1}{\beta}}(b-a)^{p+q+\frac{2}{\alpha}}}{[(2 \alpha p+1)(2 \alpha p+2)(2 \alpha q+1)(2 \alpha q+2)]^{\frac{1}{2 \alpha}}}\|h\|_{2 \beta}^{4}, & \text { if } h \in L^{2 \beta}[a, b] \\ \frac{1}{2}(b-a)^{p+q}\|h\|_{2}^{4}, & \text { if } h \in L^{2}[a, b]\end{cases}
$$

for $\alpha>1, \frac{1}{\alpha}+\frac{1}{\beta}=1$. Provided that $h(s) \neq 0$ in $[a, b]$.

Proof. From (4.11), we have

$$
\begin{aligned}
\left|\widehat{\mathcal{P}}_{h}(f, f)\right| & =\int_{a}^{b} h^{2}(x) d x \int_{a}^{b} f^{2}(x) d x-\left(\int_{a}^{b} h(x) f(x) d x\right)^{2} \\
& \leq H^{2} \begin{cases}\frac{(b-a)^{2 p+2}}{(2 p+1)(2 p+2)}\|h\|_{\infty}^{4}, & \text { if } h \in L^{\infty}[a, b] \\
\frac{2^{-\frac{1}{\beta}}(b-a)^{2 p+\frac{2}{\alpha}}}{(2 \alpha p+1)^{\frac{1}{\alpha}}(2 \alpha p+2)^{\frac{1}{\alpha}}}\|h\|_{2 \beta}^{4}, & \text { if } h \in L^{2 \beta}[a, b], \\
\frac{1}{2}(b-a)^{2 p}\|h\|_{2}^{4}, & \text { if } h \in L^{2}[a, b]\end{cases}
\end{aligned}
$$

The same inequality holds for $\left|\widehat{\mathcal{P}}_{h}(g, g)\right|$. Substituting (4.14) and that one resulting from $\left|\widehat{\mathcal{P}}_{h}(g, g)\right|$ in (4.9) we get the required result.

Corollary 9. Let $b>a>0$ and assume that $f$ and $g$ are measurable on $[a, b]$ and $\frac{f}{x}, \frac{g}{x}$ are Hölder continuous of order $p, q \in(0,1]$ with Hölderian constants $H_{1}, H_{2}>0$; respectively, 
then we have

$$
|\widehat{\mathcal{P}}(f, g)| \leq H_{1} H_{2}\left\{\begin{array}{l}
\frac{(b-a)^{p+q+2}}{\sqrt{(2 p+1)(2 p+2)(2 q+1)(2 q+2)}} b^{4}, \\
\frac{2^{-\frac{1}{\beta}}(b-a)^{p+q+\frac{2}{\alpha}}}{[(2 \alpha p+1)(2 \alpha p+2)(2 \alpha q+1)(2 \alpha q+2)]^{\frac{1}{2 \alpha}}}\left(\frac{b^{2 \beta+1}-a^{2 \beta+1}}{2 \beta+1}\right)^{\frac{2}{\beta}}, \\
\frac{1}{2}(b-a)^{p+q}\left(b^{3}-a^{3}\right)^{2},
\end{array}\right.
$$

for $\alpha>1, \frac{1}{\alpha}+\frac{1}{\beta}=1$.

Proof. Setting $h(x)=x$ in (4.13).

Theorem 9. Assume that $f$ and $g$ are measurable on $[a, b]$ and $\frac{h}{f}, \frac{h}{g}$ are Hölder continuous of order $p, q \in(0,1]$ with Hölderian constants $H_{1}, H_{2}>0$; respectively, then we have

$$
\left|\widehat{\mathcal{P}}_{h}(f, g)\right| \leq H_{1} H_{2} \begin{cases}\frac{(b-a)^{p+q+2}}{\sqrt{(2 p+1)(2 p+2)(2 q+1)(2 q+2)}}\|f\|_{\infty}^{2}\|g\|_{\infty}^{2}, & \text { if } f, g \in L^{\infty}[a, b] \\ \frac{2^{-\frac{1}{\beta}}(b-a)^{p+q+\frac{2}{\alpha}}}{[(2 \alpha p+1)(2 \alpha p+2)(2 \alpha q+1)(2 \alpha q+2)]^{\frac{1}{2 \alpha}}}\|f\|_{2 \beta}^{2}\|g\|_{2 \beta}^{2}, & \text { if } f, g \in L^{2 \beta}[a, b] \\ \frac{1}{2}(b-a)^{p+q}\|f\|_{2}^{2}\|g\|_{2}^{2}, & \text { if } f, g \in L^{2}[a, b]\end{cases}
$$

for $\alpha>1, \frac{1}{\alpha}+\frac{1}{\beta}=1$. Provided that neither $f(s)$ nor $g(s)$ equal to 0 in $[a, b]$.

Proof. From (4.11), we have

$$
\begin{aligned}
\left|\widehat{\mathcal{P}}_{h}(f, f)\right| & =\int_{a}^{b} h^{2}(x) d x \int_{a}^{b} f^{2}(x) d x-\left(\int_{a}^{b} h(x) f(x) d x\right)^{2} \\
& \leq H_{1}^{2} \begin{cases}\frac{(b-a)^{2 p+2}}{(2 p+1)(2 p+2)}\|f\|_{\infty}^{4}, & \text { if } f \in L^{\infty}[a, b] \\
\frac{2^{-\frac{1}{\beta}}(b-a)^{2 p+\frac{2}{\alpha}}}{(2 \alpha p+1)^{\frac{1}{\alpha}}(2 \alpha p+2)^{\frac{1}{\alpha}}}\|f\|_{2 \beta}^{4}, & \text { if } f \in L^{2 \beta}[a, b], \\
\frac{1}{2}(b-a)^{2 p}\|f\|_{2}^{4}, & \text { if } f \in L^{2}[a, b]\end{cases}
\end{aligned}
$$

The same inequality holds for $\left|\widehat{\mathcal{P}}_{h}(g, g)\right|$. Substituting (4.17) and that one resulting from $\left|\widehat{\mathcal{P}}_{h}(g, g)\right|$ in (4.9) we get the required result.

In [5], we find another inequality for pointwise bounded functions, which reads: If there exist constants $M \geq m>0$ such that $h(x) \geq 0$ and $M h(x) \geq f(x) \geq m h(x)$ for almost every (a.e.) $x \in[a, b]$, then

$$
\begin{aligned}
\int_{a}^{b} h^{2}(x) d x \int_{a}^{b} f^{2}(x) d x-\left(\int_{a}^{b} h(x) f(x) d x\right)^{2} & \\
& \leq \frac{1}{4} \cdot \frac{(M-m)^{2}}{m M}\left(\int_{a}^{b} h(x) f(x) d x\right)^{2} .
\end{aligned}
$$


A straight forward result regarding $\left|\widehat{\mathcal{P}}_{h}(f, g)\right|$ can be deduced as follows:

Theorem 10. If there exist constants $M \geq m>0, N \geq n>0$ such that $h(x) \geq 0$, $M h(x) \geq f(x) \geq m h(x)$ and $N h(x) \geq g(x) \geq n h(x)$ for almost every (a.e.) $x \in[a, b]$, then

$$
\left|\widehat{\mathcal{P}}_{h}(f, g)\right| \leq \frac{1}{4} \cdot \frac{(M-m)}{\sqrt{m M}} \cdot \frac{(N-n)}{\sqrt{N n}}\left|\int_{a}^{b} h(x) f(x) d x\right|\left|\int_{a}^{b} h(x) g(x) d x\right| .
$$

Proof. From (4.18), we have

$$
\begin{aligned}
\left|\widehat{\mathcal{P}}_{h}(f, f)\right| & =\int_{a}^{b} h^{2}(x) d x \int_{a}^{b} f^{2}(x) d x-\left(\int_{a}^{b} h(x) f(x) d x\right)^{2} \\
& \leq \frac{1}{4} \cdot \frac{(M-m)^{2}}{m M}\left(\int_{a}^{b} h(x) f(x) d x\right)^{2} .
\end{aligned}
$$

Similarly, for $g$ we have

$$
\begin{aligned}
\left|\widehat{\mathcal{P}}_{h}(g, g)\right| & =\int_{a}^{b} h^{2}(x) d x \int_{a}^{b} g^{2}(x) d x-\left(\int_{a}^{b} h(x) g(x) d x\right)^{2} \\
& \leq \frac{1}{4} \cdot \frac{(N-n)^{2}}{n N}\left(\int_{a}^{b} h(x) g(x) d x\right)^{2}
\end{aligned}
$$

Substituting both inequalities in the generalized pre-Grüss inequality, i.e.,

$$
\begin{aligned}
\left|\widehat{\mathcal{P}}_{h}(f, g)\right| & \leq\left|\widehat{\mathcal{P}}_{h}(f, f)\right|^{\frac{1}{2}}\left|\widehat{\mathcal{P}}_{h}(g, g)\right|^{\frac{1}{2}} \\
& \leq \frac{1}{4} \cdot \frac{(M-m)}{\sqrt{m M}} \cdot \frac{(N-n)}{\sqrt{N n}}\left|\int_{a}^{b} h(x) f(x) d x\right|\left|\int_{a}^{b} h(x) g(x) d x\right|,
\end{aligned}
$$

which completes the proof.

Remark 12. $L_{2}$-bound for $\left|\widehat{\mathcal{P}}_{h}(f, g)\right|$ can be obtained using the same approach considered in the proof of Theorem 8 which is dependent mainly on the inequality (2.18). In this case the obtained bound will be better than that one obtained in (4.13).

\section{Some RAmified ineQualities}

In this section we highlight the role of Pompeiu-Chebyshev functional $\widehat{\mathcal{P}}_{h}(\cdot, \cdot)$ in performing and obtaining new integral inequalities. Namely, by employing the functional $\widehat{\mathcal{P}}_{h}(\cdot, \cdot)$ some Hardy's type inequalities are deduced. Another inequalities for differentiable functions are considered.

5.1. Hardy-Chebyshev functional. If $f$ is nonnegative $p$-integrable $(p>1)$ function on $(0, \infty)$, then $f$ is integrable over the interval $(0, x)$ for each positive $x$ and

$$
\int_{0}^{\infty}\left(\frac{1}{x} \int_{0}^{x} f(t) d t\right)^{p} d x \leq\left(\frac{p}{p-1}\right)^{p} \int_{0}^{\infty} f^{p}(x) d x
$$

The inequality (5.1) is known in literature as Hardy Integral inequality, which was proved by Hardy in [13]. A simple and elegant proof that is closely to Hardy original ideas and appealing to Pólya's simplification that avoids technical details can be found in [15]. 
Another inequality due to Hardy [12], is that

$$
\begin{aligned}
\int_{a}^{x}\left(\frac{F(t)}{t}\right)^{2} d t & \leq 2 \int_{a}^{x} \frac{1}{t} F(t) f(t) d t \\
& \leq \int_{a}^{x}\left(\frac{F(t)}{t}\right)^{2} d t+4 \int_{x}^{2 x}\left(\frac{F(t)}{t}\right)^{2} d t
\end{aligned}
$$

where $f$ is assumed to be nonnegative and integrable function on $(a, \infty)(a>0)$ and $F(x)=\int_{a}^{x} f(t) d t$.

Almost one hundred year passed from the first result of Hardy (5.1). Through the last three decades, several applications specially in differential inequalities which play a main role in the theory of ordinary and partial differential equations have been implemented and investigated. For improvements, generalizations, extensions and useful applications of Hardy's inequality (5.1) the reader may refer to [14], [16], [17] and [18].

Next, we use Pompeiu-Chebyshev functional $\widehat{\mathcal{P}}_{h}(\cdot, \cdot)$ to study some inequalities of Hardy's type on bounded real interval $[a, b]$ under some other circumstances. The approach considered here, seems to be the first work detect or figure out the application of Chebyshev functional and its generalizations in studying Hardy type inequalities.

(1.) Let $a, b$ be positive real numbers with $a<b$. Let $f:[a, b] \rightarrow \mathbb{R}$ be an absolutely continuous on $[a, b]$. In (4.2), choose $G(x)=\frac{1}{x}, x \in[a, b]$ with $F(x)=\int_{a}^{x} f(t) d t$ for all $t \in[a, b]$ and $h=1$. Assume $F G$ is integrable on $[a, b]$ then we introduce the HardyChebyshev functional

$$
\mathcal{H}(F, G)=(b-a) \int_{a}^{b} \frac{F(t)}{t} d t-[\ln b-\ln a] \int_{a}^{b} F(t) d t
$$

or equivalently, we write $\mathcal{H}(F, G)=\widehat{\mathcal{P}}_{1}(F, G)$.

For instance, assume the assumptions of Theorem 12 are fulfilled by our choice of $F$ and $G$ as above, then we have the Hardy type inequality

$$
\int_{a}^{b} \frac{F(t)}{t} d t \geq \frac{1}{\mathcal{L}(a, b)} \cdot \int_{a}^{b} F(t) d t
$$

where $\mathcal{L}(a, b)=\frac{b-a}{\ln b-\ln a}$ is Logarithmic mean. The inequality (5.4) is sharp. Moreover, the inequality (5.4) is reversed if we applied Corollary 7 instead of Theorem 12.

On the other hand, since $\mathcal{H}(F, G)=\widehat{\mathcal{P}}_{1}(F, G)=(b-a) \mathcal{T}(F, G)$, then using the bounds in (1.2), we can state the following bounds: 
Proposition 1. Let $b>a>0$. Let $f:[a, b] \rightarrow \mathbb{R}$ be an absolutely continuous function on $[a, b]$. For $G(x)=\frac{1}{x}, x \in[a, b]$ and $F(x)=\int_{a}^{x} f(t) d t, t \in[a, b]$, we have

$$
|\mathcal{T}(F, G)| \leq \begin{cases}\frac{(b-a)^{2}}{12 a^{2}}\|f\|_{\infty}, & \text { if } f \in L_{\infty}([a, b]), \\ \frac{(b-a)^{2}}{4 a b}(M-m), & \text { if } m \leq f \leq M, \\ \frac{(b-a)^{\frac{3}{2}}}{\pi^{2}}(a b)^{-\frac{3}{2}} \mathcal{L}_{2}(a, b) \cdot\|f\|_{2}, & \text { if } f, \in L_{2}([a, b]), \\ \frac{1}{8}(b-a)\left(\frac{b-a}{a b}\right)\|f\|_{\infty}, & \text { if } f \in L_{\infty}([a, b]),\end{cases}
$$

where $L_{s}(a, b)=\left[\frac{b^{s+1}-a^{s+1}}{(s+1)(b-a)}\right]^{\frac{1}{s}}, s \in \mathbb{R} \backslash\{-1,0\}$ is the generalized Logarithmic mean.

On utilizing the pre-Grüss inequality (4.8) (with $h(t)=1$ ), we have the following result.

Proposition 2. Let $b>a>0$. Let $f:[a, b] \rightarrow \mathbb{R}$ be an integrable on $[a, b]$. Then

$$
|\mathcal{T}(F, G)| \leq\left|\frac{1}{a b}-\left(\frac{\ln b-\ln a}{b-a}\right)^{2}\right|^{\frac{1}{2}} \mathcal{T}^{\frac{1}{2}}(F, F)
$$

where

$$
\mathcal{T}(F, F)=\frac{1}{b-a} \int_{a}^{b}\left(\int_{a}^{x} f(t) d t\right)^{2} d x-\left(\frac{1}{b-a} \int_{a}^{b}\left(\int_{a}^{x} f(t) d t\right) d x\right)^{2} .
$$

The Hardy-Chebyshev functional (5.3) can be extended to be of Pompeiu-Chebyshev type as follows:

$$
\mathcal{H}_{h}(F, G)=\int_{a}^{b} h^{2}(t) d t \int_{a}^{b} \frac{F(t)}{t} d t-\int_{a}^{b} h(t) F(t) d t \int_{a}^{b} \frac{h(t)}{t} d t
$$

Clearly, $\mathcal{H}_{1}(F, G)=\mathcal{H}(F, G)$.

(2.) To generalize the previous presentations and the therefore to get more general inequalities of Hardy type, let $b>a>0$ and assume $f$ is non-negative. Therefore,

$$
\mathcal{H}\left(F^{p}, G^{p}\right)=(b-a) \int_{a}^{b}\left(\frac{F(t)}{t}\right)^{p} d t-\frac{1}{p-1} \cdot\left[\frac{a b^{p}-b a^{p}}{a^{p} b^{p}}\right] \int_{a}^{b} F^{p}(t) d t
$$

or equivalently, we write $\mathcal{H}\left(F^{p}, G^{p}\right)=\widehat{\mathcal{P}}_{1}\left(F^{p}, G^{p}\right)$, for $p>1$.

Assume the assumptions of Theorem 12 are fulfilled by our choice of $F$ and $G$ as in the previous part (1), then we have the following new inequality of Hardy's type on bounded intervals:

$$
\int_{a}^{b}\left(\frac{F(t)}{t}\right)^{p} d t \geq(a b)^{1-p} \mathcal{L}_{p}^{p}(a, b) \int_{a}^{b} F^{p}(t) d t, \quad p>1
$$

where $\mathcal{L}_{p}(a, b)=\left[\frac{b^{p-1}-a^{p-1}}{(p-1)(b-a)}\right]^{\frac{1}{p}}$ is the generalized Logarithmic mean. The inequality (5.9) is sharp. Moreover, the inequality is reversed if we applied Corollary 7 instead of Theorem 12.

On the other hand, since $\mathcal{T}\left(F^{p}, G^{p}\right)=\frac{1}{(b-a)^{2}} \widehat{\mathcal{P}}_{1}\left(F^{p}, G^{p}\right)$, then using the bounds in (1.2), we can state the following bounds: 
Proposition 3. Let $b>a>0$. Let $f:[a, b] \rightarrow[0, \infty)$ be a non-negative and absolutely continuous function on $[a, b]$. For $G(x)=\frac{1}{x}, x \in[a, b]$ and $F(x)=\int_{a}^{x} f(t) d t, t \in[a, b]$, we have

$$
\begin{aligned}
& \left|\mathcal{T}\left(F^{p}, G^{p}\right)\right| \\
& \leq \begin{cases}\frac{(b-a)^{p+1}}{12} p^{2} a^{-(p+1)}\|f\|_{\infty}^{p}, & \text { if } f \in L_{\infty}([a, b]), \\
\frac{1}{4}\left(\frac{b^{p}-a^{p}}{a^{p} b^{p}}\right)(b-a)^{p}\left(M^{p}-m^{p}\right), & \text { if } m \leq f \leq M, \\
p^{2} \frac{(b-a)^{3 / 2}}{\pi^{2}}(a b)^{-\left(p+\frac{1}{2}\right)} \mathcal{L}_{2 p}^{\frac{p}{2}}(a, b) \cdot\left\|F^{p-1} f\right\|_{2}, & \text { if } f, \in L_{2}([a, b]), \\
\frac{p}{8}(b-a)^{p}\left(\frac{b^{p}-a^{p}}{a^{p} b^{p}}\right)\|f\|_{\infty}^{p}, & \text { if } f \in L_{\infty}([a, b]),\end{cases}
\end{aligned}
$$

On utilizing the pre-Grüss inequality (4.8) (with $h(t)=1$ ), we have the following result.

Proposition 4. Let $b>a>0$. Let $f:[a, b] \rightarrow[0, \infty)$ be an integrable on $[a, b]$. Then

$$
\left|\mathcal{T}\left(F^{p}, G^{p}\right)\right| \leq\left|\frac{a b^{2 p}-b a^{2 p}}{(b-a)(2 p-1) a^{2 p} b^{2 p}}-\frac{\left(a b^{p}-b a^{p}\right)^{2}}{(b-a)^{2}(p-1)^{2} a^{2 p} b^{2 p}}\right|^{\frac{1}{2}} \mathcal{T}^{\frac{1}{2}}\left(F^{p}, F^{p}\right),
$$

for $p>1$, where

$$
\mathcal{T}\left(F^{p}, F^{p}\right)=\frac{1}{b-a} \int_{a}^{b}\left(\int_{a}^{x} f(t) d t\right)^{2 p} d x-\left(\frac{1}{b-a} \int_{a}^{b}\left(\int_{a}^{x} f(t) d t\right)^{p} d x\right)^{2} .
$$

A generalization of Hardy-Chebyshev-Pompeiu functional (5.7) can be presented such as:

$$
\mathcal{H}_{h}\left(F^{p}, G^{p}\right)=\int_{a}^{b} h^{2}(t) d t \int_{a}^{b}\left(\frac{F(t)}{t}\right)^{p} d t-\int_{a}^{b} h(t) F^{p}(t) d t \int_{a}^{b} \frac{h(t)}{t^{p}} d t, \quad p>1 .
$$

Clearly, $\mathcal{H}_{1}\left(F^{p}, G^{p}\right)=\mathcal{H}\left(F^{p}, G^{p}\right)$.

(3.) Another way to deal with Hardy-Chebyshev functional by defining the functions $\phi$ : $[a, x] \rightarrow[0, \infty), x>a \geq 0$ and $\Phi_{a}:[0, b] \rightarrow[0, \infty)$ given by $\Phi_{a}(x)=\frac{1}{x-a} \int_{a}^{x} \phi(t) d t$, $b>x>a \geq 0$. Making use of the Chebyshev functional $\mathcal{T}(\cdot, \cdot)$, we can write:

$$
\begin{aligned}
\mathcal{T}\left(\Phi_{a}, \Phi_{a}\right) & =\frac{1}{b} \int_{0}^{b} \Phi_{a}^{2}(t) d t-\left(\frac{1}{b} \int_{0}^{b} \Phi_{a}(t) d t\right)^{2} \\
& =\frac{1}{b} \int_{0}^{b}\left(\frac{1}{x-a} \int_{a}^{x} \phi(t) d t\right)^{2} d x-\left(\frac{1}{b} \int_{0}^{b}\left(\frac{1}{x-a} \int_{a}^{x} \phi(t) d t\right) d x\right)^{2} .
\end{aligned}
$$

for $p>1$. In case $a=0$, we have the Hardy functional:

$$
\mathcal{T}\left(\Phi_{0}, \Phi_{0}\right)=\frac{1}{b} \int_{0}^{b}\left(\frac{1}{x} \int_{0}^{x} \phi(t) d t\right)^{2} d x-\left(\frac{1}{b} \int_{0}^{b}\left(\frac{1}{x} \int_{0}^{x} \phi(t) d t\right) d x\right)^{2},
$$

and we have the following bound: 
Theorem 11. Let $\Phi_{0}:[0, b] \rightarrow(0, \infty)$ be such that $\Phi_{0}$ is convex on $[0, b]$. If $\Phi_{0}^{\prime} \in L_{p}[0, b]$ $1<p \leq q$, then the inequality

$$
\left|\mathcal{T}\left(\Phi_{0}, \Phi_{0}\right)\right| \leq b \frac{p q \sin \left(\frac{\pi}{p}\right) \sin \left(\frac{\pi}{q}\right)}{4 \pi^{2}(p-1)^{\frac{1}{p}}(q-1)^{\frac{1}{q}}}\left\|\Phi_{0}^{\prime}\right\|_{p}\left\|\Phi_{0}^{\prime}\right\|_{q},
$$

holds, for all $p, q>1$ with $\frac{1}{p}+\frac{1}{q}=1$.

Proof. Utilizing the triangle inequality on the right hand side of the identity

$$
\mathcal{T}\left(\Phi_{0}, \Phi_{0}\right):=\frac{1}{b} \int_{0}^{b}\left[\Phi_{0}(t)-\Phi_{0}\left(\frac{b}{2}\right)\right]\left[\Phi_{0}(t)-\frac{1}{b} \int_{0}^{b} \Phi_{0}(s) d s\right] d t
$$

and using the Hölder's inequality, we get

$$
\begin{aligned}
& \left|\mathcal{T}\left(\Phi_{0}, \Phi_{0}\right)\right| \\
& =\left|\frac{1}{b} \int_{0}^{b}\left[\Phi_{0}(t)-\Phi_{0}\left(\frac{b}{2}\right)\right]\left[\Phi_{0}(t)-\frac{1}{b} \int_{0}^{b} \Phi_{0}(s) d s\right] d t\right| \\
& \leq \frac{1}{b} \int_{0}^{b}\left|\Phi_{0}(t)-\Phi_{0}\left(\frac{b}{2}\right)\right|\left|\Phi_{0}(t)-\frac{1}{b} \int_{0}^{b} \Phi_{0}(s) d s\right| d t \\
& \leq \frac{1}{b}\left(\int_{0}^{b}\left|\Phi_{0}(t)-\Phi_{0}\left(\frac{b}{2}\right)\right|^{p} d t\right)^{1 / p} \times\left(\int_{0}^{b}\left|\Phi_{0}(t)-\frac{1}{b} \int_{a}^{b} \Phi_{0}(s) d s\right|^{q} d t\right)^{1 / q} .
\end{aligned}
$$

Since $\Phi_{0}$ is convex on $[a, b]$, then by Hermite-Hadamard inequality; i.e.,

$$
\Phi_{0}\left(\frac{b}{2}\right) \leq \frac{1}{b} \int_{a}^{b} \Phi_{0}(t) d t \leq \frac{\Phi_{0}(0)+\Phi_{0}(b)}{2},
$$

which follows that

$$
\left|\Phi_{0}(x)-\frac{1}{b} \int_{0}^{b} \Phi_{0}(t) d t\right| \leq\left|\Phi_{0}(x)-\Phi_{0}\left(\frac{b}{2}\right)\right|
$$

Now, Alomari in [4] proved that if $f$ is absolutely continuous functions whose first derivative $f^{\prime}$ is positive and $\int_{a}^{b}\left(f^{\prime}(t)\right)^{p} d t<\infty$, then for any $\xi \in(a, b)$, the inequality

$$
\int_{a}^{b}|f(t)-f(\xi)|^{p} d t \leq\left(\frac{p^{p} \sin ^{p}\left(\frac{\pi}{p}\right)}{\pi^{p}(p-1)}\right)\left[\frac{b-a}{2}+\left|\xi-\frac{a+b}{2}\right|\right]^{p} \cdot \int_{a}^{b}\left(f^{\prime}(x)\right)^{p} d x
$$

holds for all $p>1$.

Since $\Phi_{0}$ is convex and therefore is absolutely continuous on $[0, b]$ with $\Phi_{0}^{\prime} \in L_{p}[0, b]$ $(1<p<q)$, then by (5.15) we can obtain the following two inequalities which are of great interest and not less important than the main result (5.12) itself:

$$
\left(\int_{0}^{b}\left|\Phi_{0}(x)-\Phi_{0}\left(\frac{b}{2}\right)\right|^{p} d x\right)^{\frac{1}{p}} \leq \frac{p \sin \left(\frac{\pi}{p}\right)}{2 \pi(p-1)^{\frac{1}{p}}} b\left\|\Phi_{0}^{\prime}\right\|_{p}
$$


and

$$
\begin{aligned}
\left(\int_{0}^{b}\left|\Phi_{0}(x)-\frac{1}{b} \int_{0}^{b} \Phi_{0}(t) d t\right|^{q} d x\right)^{1 / q} & \leq\left(\int_{0}^{b}\left|\Phi_{0}(x)-\Phi_{0}\left(\frac{b}{2}\right)\right|^{q} d x\right)^{\frac{1}{q}} \\
& \leq \frac{q \sin \left(\frac{\pi}{q}\right)}{2 \pi(q-1)^{\frac{1}{q}}} b\left\|\Phi_{0}^{\prime}\right\|_{q},
\end{aligned}
$$

where, $p>1$ and $\frac{1}{p}+\frac{1}{q}=1$. Substituting the inequalities (5.16), (5.17) and (5.14), we get the required result (5.12).

Remark 13. The corresponding version of Hardy's inequality on bounded interval $[0, b]$ $(b>0)$, is given in the inequality (5.16) (or (5.17)). To treat this formally, we can say that: For the absolutely continuous function $\Phi_{0}:[0, b] \rightarrow(0, \infty)$ such that $\Phi_{0}^{\prime} \in L_{p}[0, b](p>1)$. If $\Phi_{0}\left(\frac{b}{2}\right)=0$ (or $\left.\int_{a}^{b} \phi(t) d t=0\right)$, then we the inequality

$$
\int_{0}^{b}\left|\Phi_{0}(x)\right|^{p} d x=\int_{0}^{b}\left(\frac{1}{x} \int_{0}^{x} \phi(t) d t\right)^{p} d x \leq \frac{p^{p} \sin ^{p}\left(\frac{\pi}{p}\right)}{2^{p} \pi^{p}(p-1)} b^{p}\left\|\Phi_{0}^{\prime}\right\|_{p}^{p} .
$$

In special case, let $b=2 \pi$ and $p=2$, then

$$
\int_{0}^{2 \pi}\left(\frac{1}{x} \int_{0}^{x} \phi(t) d t\right)^{2} d x \leq 4\left\|\Phi_{0}^{\prime}\right\|_{2}^{2}, \quad 0 \leq x \leq 2 \pi .
$$

5.2. Other inequalities. In (4.2), choose $g=\frac{1}{f}$, with $f(x)>0$, for all $x \in[a, b], f(b) \neq$ $f(a)$ and $h=f^{\prime}$ (where $f$ is assumed to be differentiable in this case), then the following identity can be ramified from (4.2)

$$
\widehat{\mathcal{P}}_{f^{\prime}}\left(f, \frac{1}{f}\right)=(b-a) \int_{a}^{b}\left(f^{\prime}(x)\right)^{2} d x-\frac{f^{2}(b)-f^{2}(a)}{2} \cdot[\ln f(b)-\ln f(a)]
$$

Thus several inequalities can be obtained for this functional.

For instance, assume the assumptions of Theorem 12 are fulfilled by our choice of $g$ and $h$ as above, then we have the new inequality

$$
\int_{a}^{b}\left(f^{\prime}(x)\right)^{2} d x \geq \frac{A(f(a), f(b))}{L(f(a), f(b))} \cdot[f(b)-f(a)],
$$

where $A(\cdot, \cdot)$ is the arithmetic mean and $L(\cdot, \cdot)$ is the Logarithmic mean. The inequality is reversed if we applied Corollary 7 instead of Theorem 12.

Also, since $f(b) \neq f(a)$, then we note that

$$
\widehat{\mathcal{P}}_{f^{\prime}}(f, f)=\left[f^{2}(b)-f^{2}(a)\right] \int_{a}^{b}\left(f^{\prime}(x)\right)^{2} d x-\frac{1}{4}\left[f^{2}(b)-f^{2}(a)\right]^{2} .
$$

For instance, applying (4.5) for (5.20), we get

$$
\int_{a}^{b}\left(f^{\prime}(x)\right)^{2} d x \geq \frac{1}{4}\left[f^{2}(b)-f^{2}(a)\right] .
$$

The inequality is reversed if we applied Corollary 7 instead of Theorem 12. 
Also, in (4.2), choose $g=f^{\prime \prime}$, with $f(x)>0$ for all $x \in[a, b]$ and $h \equiv h$ (where $f$ is assumed to be twice differentiable in this case), then the following identity can be ramified from $(4.2)$

$$
\widehat{\mathcal{P}}_{h}\left(f, f^{\prime \prime}\right)=\int_{a}^{b} h^{2}(x) d x \int_{a}^{b} f(x) f^{\prime \prime}(x) d x-\int_{a}^{b} h(t) f(t) d t \int_{a}^{b} h(t) f^{\prime \prime}(t) d t
$$

Using the results in Sections 2, 3 and 4, several inequalities can be obtained for this functional.

Among others, assume the assumptions of Theorem 12 are fulfilled by our choice of $f$ and $g$ as above, then we have the new inequality

$$
\int_{a}^{b} h^{2}(x) d x \int_{a}^{b} f(x) f^{\prime \prime}(x) d x \geq \int_{a}^{b} h(t) f(t) d t \int_{a}^{b} h(t) f^{\prime \prime}(t) d t
$$

The inequality is reversed if we applied Corollary 7 instead of Theorem 12.

Hence, by choosing any function $h$ we can state various inequalities. For example, let

(1) $h=1$, then we have

$$
\int_{a}^{b} f(x) f^{\prime \prime}(x) d x \geq \frac{f^{\prime}(b)-f^{\prime}(a)}{b-a} \int_{a}^{b} f(x) d x .
$$

(2) $h=t$, then we have

$$
\frac{b^{3}-a^{3}}{3} \int_{a}^{b} f(x) f^{\prime \prime}(x) d x \geq\{b f(b)-a f(a)-[f(b)-f(a)]\} \int_{a}^{b} x f(x) d x .
$$

(3) $h=\frac{1}{f}, f>0$, then we have

$$
\int_{a}^{b} \frac{d x}{f^{2}(x)} \int_{a}^{b} f(x) f^{\prime \prime}(x) d x \geq(b-a) \int_{a}^{b} \frac{f^{\prime \prime}(x)}{f(x)} d x .
$$

(4) $h=f^{\prime}$, then we have

$$
\int_{a}^{b}\left(f^{\prime}(x)\right)^{2} d x \int_{a}^{b} f(x) f^{\prime \prime}(x) d x \geq \frac{1}{4}\left[f^{2}(b)-f^{2}(a)\right]\left[\left(f^{\prime}(b)\right)^{2}-\left(f^{\prime}(a)\right)^{2}\right] .
$$

All inequalities are reversed if we applied Corollary 7 instead of Theorem 12.

Remark 14. The Pompeiu-Chebyshev functional $\widehat{\mathcal{P}}_{(\cdot)}(\cdot, \cdot)$ is very rich and fruitful to generate family of integral inequalities involving functions and their derivatives, as we have seen in the Section 5.

\section{REFERENCES}

[1] A.M. Acu, H. Gonska, and I. Raşa, Grüss-type and Ostrowski-type inequalities in approximation theory, Ukrainian Math. J., 636 (2011), 843-864.

[2] A.M. Acu and F.D. Sofonea, On an inequality of Ostrowski type, J. Sci. Arts, 3 (16) (2011), 281-287.

[3] A.M. Acu, A. Baboş and F.D. Sofonea, The mean value theorems and inequalities of Ostrowski type, Sci. Stud. Res. Ser. Math. Inform. 21 (1) (2011), 5-16.

[4] M.W. Alomari, On Beesack-Wirtinger inequality, Results in Mathematics, (2017). doi:10.1007/s00025016-0644-6.

[5] N.S. Barnett and S.S. Dragomir, An additive reverse of the CauchyBunyakovskySchwarz integral inequality, Appl. Math. Lett., 21 (2008), 388-393. 
[6] T. Boggio, Sur une proposition de M. Pompeiu., Mathematica (Cluj), 23 (1947), 101-102.

[7] P.L. Čebyšev, Sur les expressions approximatives des intègrals dèfinis par les outres prises entre les même limites, Proc. Math. Soc. Charkov, 2 (1882), 93-98.

[8] S.S. Dragomir, Ostrowski type inequalities for Lebesgue integral: A survey of recent results, The Australian J. Math. Anal. Appl., 14 (1) (2017), 1-287.

[9] S.S. Dragomir, Some Grüss-type results via Pompeiu's-like inequalities, Arab. J. Math. 4 (2015), 159170.

[10] S.S. Dragomir, An inequality of Ostrowski type via Pompeiu's mean value theorem, J. Inequal. Pure Appl. Math., 6 (3), (2005), Article 83.

[11] G. Grüss, Über das Maximum des absoluten Betrages von $\frac{1}{b-a} \int_{a}^{b} f(x) g(x) d x-$ $\frac{1}{(b-a)^{2}} \int_{a}^{b} f(x) d x \int_{a}^{b} g(x) d x$, Math. Z., 39 (1935), 215-226.

[12] G. H. Hardy, Notes on some points in the integral calculus, XLI. On the convergence of certain integrals and series, Messenger Math., 45 (1915), 163-166.

[13] G. H. Hardy, Note on a theorem of Hilbert, Mathematische Zeitschrift, 6 (3-4) (1920), 314-317.

[14] G. H. Hardy, J. E. Littlewood, and G. Pólya, Inequalities, 2nd ed., Cambridge University Press, Cambridge, 1967.

[15] A. Kufner, L. Maligranda and L.-E. Persson, The prehistory of the Hardy inequality, Amer. Math. Month., 113 (8) (2006), 715-732.

[16] A. Kufner, L. Maligranda, and L. E. Persson, The Hardy inequality-about its history and some related results, research report, Luleả University of Technology, Luleȧ, 2006.

[17] A. Kufner and L. E. Persson, Weighted Inequalities of Hardy Type, World Scientific, Singapore, 2003.

[18] B. Opic and A. Kufner, Hardy-Type Inequalities, Pitman Research Notes in Mathematics, no. 219, Long man Scientific \& Technical, Harlow, 1990.

[19] A. Lupaş, The best constant in an integral inequality, Mathematica, (Cluj, Romania), 15 (38) (2) (1973), 219-222.

[20] M. Matić and Š. Ungar, More on the two-point Ostrolwski inequaluty, J. Math. Ineq, 3. (3) (2009), $417-426$.

[21] G.V. Milovanović and J.E. Pečarić, On generalization of the inequality of A. Ostrowski and some related applications, Univ. Beograd. Publ. Elektrotehn. Fak. Ser. Mat. Fiz., 544-576 (1976), 155-158

[22] D.S. Mitrinović, J.E. Pečarić and A.M. Fink, Classical and New Inequalities in Analysis, in: Mathematics and its Applications (East European Series), vol. 61, Kluwer Academic Publishers Group, Dordrecht 1993.

[23] A.M. Ostrowski, On an integral inequality, Aequat. Math., 4 (1970), 358-373.

[24] B.G. Pachpatte, On Grüss like integral inequalities via Pompeiu's mean value theorem, J. Inequal. Pure Appl. Math., 6 (3), (2005), Article 82.

[25] J. Pečarić and Š. Ungar, On an inequality of Ostrowski type, J. Inequal. Pure Appl. Math. 7 (4), (2006), Article 151.

[26] D. Pompeiu, Sur une proposition analogue au theoreme des accroissements finis, Mathematica (Cluj), 22 (1946), 143-146.

[27] E.C. Popa, An inequality of Ostrowski type via a mean value theorem, Gen. Math., 15 (1) (2007), 93-100.

[28] P. Sahoo and T. Riedel, Mean Value Theorems and Functional Equations, Singapore, World Scientific Publishing Co. Pte. Ltd. 1998.

Department of Mathematics, Faculty of Science and Information Technology, Irbid National University, P.O. Box 2600, Irbid, P.C. 21110, Jordan.

E-mail address: mwomath@gmail.com 